\title{
ATTRACTORS OF THE REACTION-DIFFUSION SYSTEMS WITH RAPIDLY OSCILLATING COEFFICIENTS AND THEIR HOMOGENIZATION
}

\section{ATTRACTEURS POUR DES SYSTÉMES DE RÉACTION-DIFFUSION AVEC DES COEFFICIENTS OSCILLANT RAPIDEMENT ET LEUR HOMOGÉNÉISATION}

\author{
M. EFENDIEV ${ }^{\text {a }}$, S. ZELIK ${ }^{\text {b }}$ \\ ${ }^{\text {a } W e i e r s t r a s s ~ I n s t i t u t e ~ f o r ~ A p p l i e d ~ M a t h e m a t i c s ~ a n d ~ S t o c h a s t i c s, ~ M o h r e n s t r a s s e ~ 39, ~}$ \\ 10117, Berlin, Germany \\ ${ }^{\mathrm{b}}$ Institute for Problems of Transmission Information, Russian Academy of Sciences, Bolshoj Karetnij 19, \\ Moscow, 101447, GSP-4, Russia
}

Received 10 July 2000, revised 7 May 2001

Dedicated to Prof. W. Jäger on the occasion of his 60th birthday

ABSTRACT. - The following reaction-diffusion system in spatially non-homogeneous almostperiodic media is considered in a bounded domain $\Omega \subset \mathbb{R}^{3}$ :

$$
\partial_{t} u=A_{\varepsilon} u-f(u)+g,\left.\quad u\right|_{\partial \Omega}=0 .
$$

Here $u=\left(u^{1}, \ldots, u^{k}\right)$ is an unknown vector-valued function, $f$ is a given nonlinear interaction function and the second order elliptic operator $A_{\varepsilon}$ has the following structure:

$$
\left(A_{\varepsilon} u\right)^{l}=\sum_{i, j=1}^{3} \partial_{x_{i}}\left(a_{i j}^{l}\left(\varepsilon^{-1} x\right) \partial_{x_{j}} u^{l}(x)\right), \quad \varepsilon \ll 1, l=1,2,3,
$$

where $a_{i j}^{l}(y)$ are given almost-periodic functions. We prove that, under natural assumptions on the nonlinear term $f(u)$, the longtime behavior of solutions of (1) can be described in terms of the global attractor $\mathcal{A}^{\varepsilon}$ of the associated dynamical system and that the attractors $\mathcal{A}^{\varepsilon}, 0<\varepsilon<\varepsilon_{0} \ll 1$, converge to the attractor $\mathcal{A}^{0}$ of the homogenized problem (1) as $\varepsilon \rightarrow 0$. Moreover, in the 
particular case of periodic media, we give explicit estimates for the distance between the nonhomogenized $\mathcal{A}^{\varepsilon}$ and the homogenized $\mathcal{A}^{0}$ attractors in terms of the parameter $\varepsilon$.

(C) 2002 L'Association Publications de l'Institut Henri Poincaré. Published by Elsevier B.V. All rights reserved

1991 MSC: 35L30; 35B40; 35B45

RÉSUMÉ. - Nous considérons le systéme de réaction-diffusion suivant dans un milieu non homogéne presque périodique en espace dans un domaine borné $\Omega \subset \mathbb{R}^{3}$ :

$$
\partial_{t} u=A_{\varepsilon} u-f(u)+g,\left.\quad u\right|_{\partial \Omega}=0 .
$$

Ici, $u=\left(u^{1}, \ldots, u^{k}\right)$ est une fonction vectorielle inconnue, $f$ est une fonction d'interaction nonlinéaire donnée et l'opérateur elliptique du second ordre $A_{\varepsilon}$ a la structure suivante :

$$
\left(A_{\varepsilon} u\right)^{l}=\sum_{i, j=1}^{3} \partial_{x_{i}}\left(a_{i j}^{l}\left(\varepsilon^{-1} x\right) \partial_{x_{j}} u^{l}(x)\right), \quad \varepsilon \ll 1, l=1,2,3,
$$

oú les $a_{i j}^{l}(y)$ sont des fonctions presque périodiques données. Nous prouvons que, sous des hypothéses naturelles sur le term nonlinéaire $f(u)$, le comportement asymptotique des solutions de (1) peut etre décrit par l'attracteur global $\mathcal{A}^{\varepsilon}$ du systéme dynamique associé et que les attracteurs $\mathcal{A}^{\varepsilon}, 0<\varepsilon<\varepsilon_{0} \ll 1$, convergent vers l'attracteur $\mathcal{A}^{0}$ du probléme homogénéisé de (1) lorsque $\varepsilon \rightarrow 0$. De pluis, dans le cas particulier d'un milieu périodique, nous donnons des estimations explicites de la distance entre les attracteur non homogénéisés $\mathcal{A}^{\varepsilon}$ et l'attracteur homogénéisé $\mathcal{A}^{0}$ en fonction du paramétre $\varepsilon$.

(C) 2002 L'Association Publications de l'Institut Henri Poincaré. Published by Elsevier B.V. All rights reserved

\section{Introduction}

We consider the reaction-diffusion system

$$
\left\{\begin{array}{l}
\partial_{t} u=A_{\varepsilon} u-f(u)+g, \quad x \in \Omega, \\
\left.u\right|_{t=0}=u_{0},\left.\quad u\right|_{\partial \Omega}=0
\end{array}\right.
$$

in a bounded domain $\Omega \Subset \mathbb{R}^{3}$ with a sufficiently smooth boundary. Here $u=$ $u(t, x)=\left(u^{1}, \ldots, u^{k}\right)$ is an unknown vector-valued function, the functions $f(u)=$ $\left(f^{1}(u), \ldots, f^{k}(u)\right)$ and $g=g(x)=\left(g^{1}, \ldots, g^{k}\right)$ are given and the second order elliptic differential operator $A_{\varepsilon}$ has the following form:

$$
A_{\varepsilon} u:=\operatorname{diag}\left(A_{\varepsilon}^{1} u^{1}, \ldots, A_{\varepsilon}^{k} u^{k}\right)
$$

with

$$
A_{\varepsilon}^{l} u^{l}:=\sum_{i, j=1}^{3} \partial_{x_{i}}\left(a_{i j}^{l}\left(\varepsilon^{-1} x\right) \partial_{x_{j}} u^{l}(x)\right), \quad \varepsilon \ll 1,
$$

where the functions $a_{i j}^{l}(y), y \in \mathbb{R}^{3}$ are assumed to be symmetric $\left(a_{i j}^{l}(y) \equiv a_{j i}^{l}(y)\right)$ and almost-periodic with respect to $y \in \mathbb{R}^{3}$ (i.e. $a_{i j}^{l} \in A P\left(\mathbb{R}^{3}\right)$ see e.g. [10]) for every fixed 
indexes $i, j, l$ and the uniform ellipticity condition

$$
\sum_{i, j} a_{i j}^{l}(y) \xi_{i} \xi_{j} \geqslant v|\xi|^{2}, \quad y, \xi \in \mathbb{R}^{3}
$$

is also assumed (with the appropriate $v>0$ ) to be valid for every operator $A_{\varepsilon}^{l}$.

We impose to the nonlinear term $f$ the following regularity conditions

$$
\left\{\begin{array}{l}
\text { 1. } f \in C^{1}\left(\mathbb{R}^{k}, \mathbb{R}^{k}\right) \\
\text { 2. }|f(u)| \leqslant C\left(1+|u|^{p}\right),
\end{array}\right.
$$

for a certain $p \geqslant 1$, and the following anisotropic dissipativity condition: there are the exponents $p_{i} \geqslant 2(p-1), i=1, \ldots, k$, such that

$$
\sum_{i=1}^{k} f_{i}(v) v^{i}\left|v^{i}\right|^{p_{i}} \geqslant-C, \quad \text { for all } v \in \mathbb{R}^{k}
$$

which generalizes the standard isotropic one $\left(p_{i}=0\right)$ and from the ours point of view is more adopted to study the problems of type (0.1) with diagonal leading part $A_{\varepsilon}$.

For example, if $k=2, u=(v, w)$ the anisotropic dissipativity assumption is satisfied for the following non-linearities:

$$
f(u)=\left(\begin{array}{c}
v^{3}-\alpha v-\beta w \\
w-\gamma v
\end{array}\right), \quad \text { or } \quad f(u)=\left(\begin{array}{c}
v^{3}-w \\
w-v^{3}
\end{array}\right), \quad \alpha, \beta, \gamma \in \mathbb{R} .
$$

Note that the first example (which corresponds to the Fitz-Nagumo equation see [8]) satisfies the standard isotropic dissipativity assumption as well; the second nonlinearity satisfies the relation $f^{1}(u)+f^{2}(u)=0$ (which is natural for chemical kinetics) and evidently does not satisfy the isotropic dissipativity condition (see the end of Section 2 for the further examples).

It is assumed also that the external force $g \in L^{2}(\Omega)$, the initial date $u_{0} \in L^{\infty}(\Omega)$, and the solution $u(t)$ of problem $(0.1)$ is defined to be a function

$$
u \in L^{\infty}([0, T] \times \Omega) \cap L^{2}\left([0, T], W_{0}^{1,2}(\Omega)\right) \cap C\left([0, T], L^{2}(\Omega)\right)
$$

which satisfies Eq. (0.1) in the sense of distributions. (Here and below, we denote by $W^{l, p}$ the Sobolev space of functions whose derivatives up to order $l$ inclusively belong to $L^{p}$ and $\|\cdot\|_{l, p}:=\|\cdot\|_{W^{l, p}}$.)

The problems of type $(0.1)$ has been intensively studied by many authors. The longtime behaviour of solutions of (0.1) for fixed $\varepsilon>0$ are considered under the various assumptions on the nonlinear term $f$ and the operator $A_{\varepsilon}=A$ in $[1,6,13]$.

The homogenization problems for individual solutions of linear and nonlinear elliptic or parabolic equations of the form $(0.1)$ has been investigated in $[2,5,7,20]$ (see also the references therein).

The longtime behaviour of solutions of RDE and even hyperbolic equations in the nonhomogenized periodic media with asymptotic degeneracy has been studied in $[3,12]$. 
In the present paper, we study the case where we have a system of reaction-diffusion equations $(0.1)$ in the non-homogenized almost-periodic media. (For simplicity, we restrict ourselves to consider only the most relevant from the applications point of view 3-dimensional case, but the applied methods work after the minor changings for an arbitrary space dimension $n$.)

It is proved that, under assumptions (0.2)-(0.6), problem (0.1) possesses a global attractor $\mathcal{A}^{\varepsilon}$ in the phase space $u_{0} \in \Phi=L^{\infty}(\Omega)$, for every fixed $\varepsilon>0$. Moreover, these attractors are occured to be uniformly bounded with respect to $\varepsilon \rightarrow 0$ in the space $C^{2 \beta}(\Omega)$ of Hölder continuous functions with an appropriate Holder exponent $1>2 \beta>0$ :

$$
\left\|\mathcal{A}^{\varepsilon}\right\|_{C^{2 \beta}(\Omega)} \leqslant C \text { for } \varepsilon \leqslant \varepsilon_{0} .
$$

In order to study the behaviour of attractors $\mathcal{A}^{\varepsilon}$ when $\varepsilon \rightarrow 0$, we introduce the homogenized problem for equation $(0.1)$

$$
\left\{\begin{array}{l}
\partial_{t} u=A_{0} u-f(u)+g \\
\left.u\right|_{\partial \Omega}=0,\left.\quad u\right|_{t=0}=u_{0}
\end{array}\right.
$$

where $A_{0}=\operatorname{diag}\left\{A_{0}^{1}, \ldots, A_{0}^{k}\right\}$ and the elliptic operators $A_{0}^{l}$ are constructed by the standard formulae of the almost-periodic homogenization theory (see e.g. [20]).

It is proved that this limit problem also possesses the attractor $\mathcal{A}^{0}$ in the phase space $u_{0} \in \Phi$ and the family of attractors $\mathcal{A}^{\varepsilon}$ tends to the limit attractor $\mathcal{A}^{0}$ in the following sense:

$$
\operatorname{dist}_{C^{2 \beta^{\prime}}(\Omega)}\left(\mathcal{A}^{\varepsilon}, \mathcal{A}^{0}\right) \rightarrow 0 \quad \text { when } \varepsilon \rightarrow 0 .
$$

Here the Hölder exponent $0<2 \beta^{\prime}<2 \beta<1$ and $\operatorname{dist}_{V}$ denotes the non-symmetric Hausdorff distance between subsets of the space $V$ :

$$
\operatorname{dist}_{V}(X, Y):=\sup _{x \in X} \inf _{y \in Y}\|x-y\|_{V} .
$$

Thus, the attractors $\mathcal{A}^{\varepsilon}, \varepsilon \in\left[0, \varepsilon_{0}\right]$, are occured to be upper-semicontinious at $\varepsilon=0$ in the space $C^{2 \beta^{\prime}}(\Omega)$.

In order to illustrate the obtained results, we give a more detailed consideration of the case where the coefficients $a_{i j}^{l}$ are assumed to be periodic. In this case, imposing some additional requirements on the structure of the limit attractor $\mathcal{A}^{0}$ and using the method of asymptotic expansions, we give the explicit estimate of the error of approximation of the non-homogenized attractors of $(0.1)$ by the attractor $\mathcal{A}^{0}$ of the homogenized equation (0.9). Namely, assume in addition that the limit attractor is exponential, i.e. there is a positive number $v>0$ and the function $Q$ such that, for every bounded subset $B \subset \Phi$, the following is true:

$$
\operatorname{dist}_{L^{2}(\Omega)}\left(S_{t}^{0} B, \mathcal{A}^{0}\right) \leqslant Q\left(\|B\|_{\Phi}\right) \mathrm{e}^{-v t},
$$

where $S_{t}^{0}$ is a semigroup in $\Phi$ generated by the limit equation (0.9). 
THEOREM. - Let the coefficients $a_{i j}^{l}$ be periodic and smooth enough and let the limit attractor $\mathcal{A}^{0}$ be exponential. Then

$$
\operatorname{dist}_{C(\Omega)}\left(\mathcal{A}^{\varepsilon}, \mathcal{A}^{0}\right) \leqslant C \varepsilon^{\kappa},
$$

where the constant $C$ and exponent $0<\kappa<1$ can be calculated explicitly.

It is worth to emphasize also that the exponential rate of convergence $(0.12)$ has been already established for some classes of equations (0.9). For instance, let the nonlinear term $f(u)$ be potential, i.e.

$$
f(u)=\nabla_{u} F(u), \quad F \in C^{2}\left(\mathbb{R}^{k}, \mathbb{R}\right)
$$

which is always true in a scalar case $k=1$. Then, Eq. (0.9) evidently possesses the global Lyapunov function and, consequently, for generic $g$ 's (for which equation (0.9) has a finite number of equilibria and all of them are hyperbolic), the attractor $\mathcal{A}^{0}$ is regular and exponential (see $[1,15])$. Therefore, due to the theorem, the estimate $(0.13)$ is valid in this case.

Note that the estimates of the form (0.13) (for a symmetric or non-symmetric distance) for the regular attractors of the abstract semigroups which possess the Lyapunov functions and depend regularly on a parameter $\varepsilon$ has been obtained in [1] (see Remark 4.3 for estimate (0.13) of the symmetric distance in our case).

These results has been recently applied in [16] for obtaining the estimates of the form (0.13) for the reaction diffusion equations with $A_{\varepsilon} \equiv \Delta_{x}$ and spatial rapid quasiperiodic oscilations in the subbordinated terms (i.e. $f(u):=a\left(\varepsilon^{-1} x\right) \tilde{f}(u)$ or a more general non-linearities $f\left(u, \varepsilon^{-1} x\right)$ ). The analogues of these estimates for a singular perturbed non-autonomous parabolic systems with rapid temporal oscilations in subbordinated terms (for instance with $g=g\left(\varepsilon^{-1} t, x\right)$ ) has been obtained in [19].

\section{Uniform a priori estimates}

In this section, we derive several estimates for the solutions of Eq. (0.1) which are of fundamental significance for our purposes.

We start with the uniform (with respect to $\varepsilon \rightarrow 0$ ) $L^{p_{i}}$-estimates for the solutions of (0.1).

THEOREM 1.1. - Let assumptions (0.2)-(0.6) be satisfied and let $u$ be a solution of problem (0.1). Then, the following estimate is valid:

$$
\sum_{i=1}^{k}\left\|u^{i}(t)\right\|_{0, p_{i}+2}^{p_{i}+2} \leqslant C\left(\sum_{i=1}^{k}\left\|u^{i}(0)\right\|_{0, p_{i}+2}^{p_{i}+2}\right) \mathrm{e}^{-\alpha t}+C\left(1+\sum_{i=1}^{k}\|g\|_{0,2}^{p_{i}+2}\right),
$$

where $C, \alpha>0$ are independent of $\varepsilon$.

Proof. - Let us multiply the $l$ th equation of $(0.1)$ by $u^{l}(t)\left|u^{l}(t)\right|^{p_{l}}$, integrate over $x \in \Omega$ and integrate by parts the leading term $A_{\varepsilon}^{l}$ in the following way: 


$$
\begin{aligned}
\left(A_{\varepsilon}^{l} u^{l}, u^{l}\left|u^{l}\right|^{p_{l}}\right) & =-\frac{4\left(p_{l}+1\right)}{p_{l}^{2}}\left(\sum_{i, j} a_{i j}^{l} \partial_{x_{j}}\left(\left|u^{l}\right|^{\left(p_{l}+2\right) / 2}\right), \partial_{x_{i}}\left(\left|u^{l}\right|^{\left(p_{l}+2\right) / 2}\right)\right) \\
& \leqslant-C v\left\|\nabla_{x}\left(\left|u^{l}\right|^{\left(p_{l}+2\right) / 2}\right)\right\|_{0,2}^{2}
\end{aligned}
$$

(here we have used also the uniform ellipticity (0.4)).

Taking the sum of all obtained inequalities and taking into the account the dissipativity assumption (0.6), we obtain

$$
\begin{gathered}
\partial_{t}\left(\sum_{i=1}^{k}\left\|u^{i}(t)\right\|_{0, p_{i}+2}^{p_{i}+2}\right)+C v \sum_{i=1}^{k}\left\|\nabla_{x}\left(\left|u^{i}(t)\right|^{\left(p_{i}+2\right) / 2}\right)\right\|_{0,2}^{2} \\
\leqslant C_{1}+\sum_{i=1}^{k}\left(g^{i}, u^{i}(t)\left|u^{i}(t)\right|^{p_{i}}\right) \equiv C_{1}+G_{u}(t) .
\end{gathered}
$$

Denote also

$$
F_{u}(t):=\sum_{i=1}^{k}\left\|u^{i}(t)\right\|_{0, p_{i}+2}^{p_{i}+2}, \quad \Phi_{u}(t):=\sum_{i=1}^{k}\left\|\nabla_{x}\left(\left|u^{i}(t)\right|^{\left(p_{l}+2\right) / 2}\right)\right\|_{0,2}^{2} .
$$

We now note that, due to the Fridrich's inequality, $F_{u}(t) \leqslant C_{1} \Phi_{u}(t)$ and, consequently, applying the Gronwall inequality to (1.3), we derive after the standard computations that

$$
\begin{aligned}
& \sup _{t \in[T, T+1]} F_{u}(t)+\int_{T}^{T+1} \Phi_{u}(t) \mathrm{d} t \\
& \leqslant C F_{u}(0) \mathrm{e}^{-\alpha T}+C_{1}+\int_{0}^{T+1} \mathrm{e}^{-\alpha(T+1-t)}\left|G_{u}(t)\right| \mathrm{d} t,
\end{aligned}
$$

with the appropriate $\alpha>0$.

Thus, the main problem now is to estimate the integral into the right-hand side of (1.5). To this end, we transform it to the following form which is more convenient for our purposes:

$$
\int_{0}^{T+1}\left|G_{u}(t)\right| \mathrm{d} t \leqslant C_{2} \sup _{t \in[0, T]}\left(\mathrm{e}^{-\alpha(t-T) / 2} \int_{t}^{t+1} \mathrm{e}^{-\alpha(T+1-t)}\left|G_{u}(s)\right| \mathrm{d} s\right) .
$$

It is essential for us that the constant $C_{2}$ is independent of $T$.

Let $W_{i}([t, t+1]):=L^{p_{i}+1}\left([t, t+1], L^{2\left(p_{i}+1\right)}(\Omega)\right)$. Then, due to Hölder inequality

$$
\int_{t}^{t+1}\left|\left(g^{i}, u^{i}(s)\left|u^{i}(s)\right|^{p_{i}}\right)\right| \mathrm{d} s \leqslant\left\|u^{i}\right\|_{W_{i}([t, t+1])}^{p_{i}+1}\|g\|_{0,2} \leqslant \mu\left\|u^{i}\right\|_{W_{i}([t, t+1])}^{p_{i}+2}+C_{\mu}\|g\|_{0,2}^{p_{i}+2},
$$

where the constant $\mu$ can be chosen arbitrarily small. In order to estimate the first term in the left-hand side of (1.7), we need the following interpolation result. 
Lemma 1.1. - Let $\Omega \Subset \mathbb{R}^{3}$ be smooth domain. Then

$$
\|v\|_{L^{q}\left([t, t+1], L^{2 q}(\Omega)\right)} \leqslant C\|v\|_{L^{\infty}\left([t, t+1], L^{2}(\Omega)\right)}^{1-\theta}\|v\|_{L^{2}\left([t, t+1], W^{1,2}(\Omega)\right)}^{\theta},
$$

where $q=\frac{7}{3}$ and $\theta=\frac{6}{7}$.

Proof. - Indeed, according to the standard interplolation theorem (see e.g. [14]), inequality (1.8) is valid with exponents $q$ and $\theta$ satisfying the relations

$$
\frac{1}{q}=\frac{1-\theta}{\infty}+\frac{\theta}{2} ; \quad \frac{1}{2 q}=\frac{1-\theta}{2}+\theta\left(\frac{1}{2}-\frac{1}{3}\right) .
$$

Solving system (1.9), we obtain the exponents from the lemma. Lemma 1.1 is proved.

Embedding (1.8) implies, particularly, that

$$
\begin{aligned}
\left\|u^{i}\right\|_{W_{i}([t, t+1])}^{p_{i}+2} & \leqslant C\left\|u^{i}\right\|_{L^{q\left(p_{i}+2\right) / 2}\left([t, t+1], L^{q\left(p_{i}+2\right)}(\Omega)\right)}^{p_{i}+2} \\
& \leqslant C_{1}\left(\sup _{s \in[t, t+1]}\left\|u^{i}(s)\right\|_{0, p_{i}+2}^{p_{i}+2}+\int_{t}^{t+1}\left\|\nabla_{x}\left(\left|u^{i}(s)\right|^{\left(p_{i}+2\right) / 2}\right)\right\|_{0,2}^{2} \mathrm{~d} s\right) \\
& \leqslant C_{2}\left(\sup _{s \in[t, t+1]} F_{u}(s)+\int_{t}^{t+1} \Phi_{u}(s) \mathrm{d} s\right)
\end{aligned}
$$

(here we have used the evident fact that $\left.q\left(p_{i}+2\right) / 2=\frac{7}{6}\left(p_{i}+2\right)>p_{i}+1\right)$.

Inserting this estimate to the right-hand side of (1.7) and taking a sum over the indexes $i=1, \ldots, k$, we derive the estimate

$$
\int_{t}^{t+1}\left|G_{u}(s)\right| \mathrm{d} s \leqslant \mu\left(\sup _{s \in[t, t+1]} F_{u}(s)+\int_{t}^{t+1} \Phi_{u}(s) \mathrm{d} s\right)+C_{\mu} \sum_{i=1}^{k}\|g\|_{0,2}^{p_{i}+2},
$$

where $\mu>0$ can be chosen arbitrarily small.

Denoting the left-hand side of (1.5) by $Z_{u}(T)$ and inserting estimate (1.11) into the right-hand side of (1.5), we have

$$
Z_{u}(T) \leqslant C F_{u}(0) \mathrm{e}^{-\alpha T}+\mu \sup _{t \in[0, T]}\left(\mathrm{e}^{-\alpha(T-t) / 2} Z_{u}(t)\right)+C_{\mu}\left(\sum_{i=1}^{k}\|g\|_{0,2}^{p_{i}+2}+1\right) .
$$

It can be easily proved (see e.g. [17]) that, for $\mu<1 / 2$, (1.12) implies the estimate

$$
Z_{u}(T) \leqslant C_{1} F_{u}(0) \mathrm{e}^{-\alpha T / 2}+C_{2}\left(\sum_{i=1}^{k}\|g\|_{0,2}^{p_{i}+2}+1\right) .
$$

Theorem 1.1 is proved. 
COROLlaRY 1.1. - Let the assumptions of Theorem 1.1 hold. Then

$$
\|f(u(t))\|_{0,2} \leqslant Q\left(\left\|u_{0}\right\|_{0, \infty}\right) \mathrm{e}^{-\alpha t}+Q\left(\|g\|_{0,2}\right),
$$

for the appropriate $\alpha>0$ and monotonic function $Q$ which are independent of $\varepsilon$.

Indeed, (1.14) is an immediate corollary of (1.1), the second assumption of (0.5) and our choice of the exponents $p_{i}\left(2 p \leqslant p_{i}+2\right)$.

Remark 1.1. - Note that estimate (1.14) (and consequently all corollaries of it which will be formulated below) remains valid if we replace the growth restriction 2 ) from $(0.5)$ by the following one:

$$
|f(v)|^{2} \leqslant C\left(1+\sum_{i=1}^{k}\left|v^{i}\right|^{p_{i}+2}\right) .
$$

Having the uniform estimate (1.14) for the nonlinear term $f(u)$ in Eq. (0.1), we can apply the standard methods (see e.g. [1,9]) in order to obtain more convenient estimates. Since these methods are well known, we formulate below the estimates and indicate only the main ideas of their proofs (in order to show that they are really uniform with respect to $\varepsilon$ ).

Firstly, taking the inner product in $\mathbb{R}^{k}$ of Eq. (0.1) with the function $u(t)$, integrating by $x \in \Omega$ (with the integration by parts in $A_{\varepsilon}$ and using the uniform ellipticity conditions), estimating the non-linear term by (1.14) and applying the Gronwall inequality, we obtain the following estimate.

PROPOSITION 1.1. - Let the above assumptions hold. Then

$$
\|u(T)\|_{0,2}^{2}+\int_{T}^{T+1}\|u(t)\|_{1,2}^{2} \mathrm{~d} t \leqslant Q\left(\left\|u_{0}\right\|_{0, \infty}\right) \mathrm{e}^{-\alpha T}+Q\left(\|g\|_{0,2}\right),
$$

where the constant $\alpha>0$ and the monotonic function $Q$ (which are not the same as in (1.14)) are independent of $\varepsilon$.

COROLLARY 1.2. - Let the above assumptions hold. Then

$$
\begin{gathered}
\|u(T)\|_{1,2}^{2}+\int_{T}^{T+1}\left\|A_{\varepsilon} u(t)\right\|_{0,2}^{2} \mathrm{~d} t+\int_{T}^{T+1}\left\|\partial_{t} u(t)\right\|_{0,2}^{2} \mathrm{~d} t \\
\leqslant\left(Q\left(\left\|u_{0}\right\|_{0, \infty}\right)+C\left\|u_{0}\right\|_{1,2}^{2}\right) \mathrm{e}^{-\alpha T}+Q\left(\|g\|_{0,2}\right),
\end{gathered}
$$

where the constants $\alpha, C>0$ and the function $Q$ are independent of $\varepsilon$.

Proof. - Indeed, taking the scalar product in $\mathbb{R}^{k}$ of Eq. (0.1) with the function $A_{\varepsilon} u(t)$, integrating by parts the term $\left(\partial_{t} u, A_{\varepsilon} u\right)$ and using the fact that $A_{\varepsilon}^{l}$ are symmetric, we obtain the inequality

$$
\partial_{t}\left(\sum_{i, j, l} a_{i j}^{l} \partial_{i} u^{l}(t), \partial_{j} u^{l}(t)\right)+\left(A_{\varepsilon} u^{l}(t), A_{\varepsilon} u^{l}(t)\right)+\sum_{i, j, l}\left(a_{i j}^{l} \partial_{i} u^{l}(t), \partial_{j} u^{l}(t)\right)
$$




$$
\leqslant \sum_{i, j, l}\left(a_{i j}^{l} \partial_{i} u^{l}(t), \partial_{j} u^{l}(t)\right)+C\left(\|f(u(t))\|_{0,2}^{2}+\|g\|_{0,2}^{2}\right) .
$$

Recall now that the coefficients $a_{i j}^{l}(y)$ are assumed to be almost-periodic and, consequently, uniformly bounded in $\mathbb{R}^{3}$. Therefore, the first term in the right-hand side of (1.18) can be estimated by $C_{a}\|u(t)\|_{1,2}^{2}$ where the constant $C_{a}$ is independent of $\varepsilon$. Inserting now estimate (1.14) into the right-hand side of (1.18), applying the Gronwall inequality and using (1.16) for estimating the integral of the $W^{1,2}$-norm, we derive estimate (1.17), but without the norm of $\partial_{t} u$ in the left-hand side:

$$
\|u(T)\|_{1,2}^{2}+\int_{T}^{T+1}\left\|A_{\varepsilon} u(t)\right\|_{0,2}^{2} \mathrm{~d} t \leqslant\left(Q\left(\left\|u_{0}\right\|_{0, \infty}\right)+C\left\|u_{0}\right\|_{1,2}^{2}\right) \mathrm{e}^{-\alpha t}+Q\left(\|g\|_{0,2}\right) .
$$

The estimate for the time derivative $\partial_{t} u$ can be easily obtained now from equation (0.1) and from estimates (1.14) and (1.19). Corollary 1.2 is proved.

Analogously, taking the inner product of (0.1) with the function $t A_{\varepsilon}^{l} u^{l}(t)$, we derive in a standard way the following version of the smoothing property.

COROLLARY 1.3. - Let the above assumptions hold. Then

$$
\begin{gathered}
\|u(T)\|_{1,2}^{2}+\int_{T}^{T+1}\left\|\partial_{t} u(t)\right\|_{0,2}^{2} \mathrm{~d} t+\int_{T}^{T+1}\left\|A_{\varepsilon} u(t)\right\|_{0,2}^{2} \mathrm{~d} t \\
\leqslant \frac{T+1}{T}\left(Q\left(\left\|u_{0}\right\|_{0, \infty}\right) \mathrm{e}^{-\alpha T}+Q\left(\|g\|_{0,2}\right)\right),
\end{gathered}
$$

where $\alpha>0$ and $Q$ are independent of $\varepsilon>0$.

COROLLARY 1.4. - Let the previous assumptions be valid. Then, the following estimate holds:

$$
\|u(t)\|_{0, \infty} \leqslant Q\left(\left\|u_{0}\right\|_{0, \infty}\right) \mathrm{e}^{-\alpha t}+Q\left(\|g\|_{0,2}\right),
$$

where the monotonic function $Q$ and the constant $\alpha>0$ are independent of $\varepsilon$. Moreover, there is a positive number $\beta>0$ (independent of $\varepsilon$ ) such that, for every $T \geqslant 1$, the solutions $u(t)$ of $(0.1)$ belong to the space $C^{\beta, 2 \beta}([T, T+1] \times \Omega)$ of Hölder continuous functions with Hölder constants $\beta$ and $2 \beta$ with respect to the variables $t$ and $x$ correspondingly (see [9]) and the following estimate is valid:

$$
\|u\|_{C^{\beta, 2 \beta}([T, T+1] \times \Omega)} \leqslant Q_{1}\left(\left\|u_{0}\right\|_{0, \infty}\right) \mathrm{e}^{-\alpha T}+Q_{1}\left(\|g\|_{0,2}\right), \quad T \geqslant 1,
$$

where the constant $\alpha$ and the function $Q_{1}$ are independent of $\varepsilon$.

Proof. - Indeed, let us consider the $l$ th equation of $(0.1)$

$$
\partial_{t} u^{l}-A_{\varepsilon}^{l} u^{l}=f^{l}(u(t))+g^{l}:=h^{l}(t) .
$$

Applying the maximum principle for solutions from class (0.7) of this equation (see [9, Theorem 3.7.1]), we obtain that, for $t \in[0, T]$ 


$$
\begin{aligned}
\left\|u^{l}(t)\right\|_{0, \infty} & \leqslant C\left(\left\|u_{0}^{l}\right\|_{0, \infty}+\left\|h^{l}\right\|_{L^{\infty}\left([0, T], L^{2}(\Omega)\right)}\right) \\
& \leqslant C\|g\|_{0,2}+C\|f(u)\|_{L^{\infty}\left([0, T], L^{2}(\Omega)\right)}
\end{aligned}
$$

where the constant $C$ depends only on the $L^{\infty}$-norm of $a_{i j}^{l}$ and on the uniform ellipticity constant (0.4) (and is independent of $\varepsilon$ ).

Inserting estimate (1.14) into the rigth-hand side of (1.24), we deduce that

$$
\|u(t)\|_{0, \infty} \leqslant Q\left(\left\|u_{0}\right\|_{0, \infty}\right)+Q\left(\|g\|_{0,2}\right) .
$$

Note, however, that estimate (1.25) is not dissipative with respect to the initial conditions $u_{0}$. In order to obtain the dissipative one, we fix $T \geqslant 1$ and consider the function $v^{l}(t)=(t-T+1) u^{l}(t)$ which evidently satisfies the equation

$$
\begin{aligned}
& \partial_{t} v^{l}-A^{\varepsilon} v^{l}=u^{l}(t)+(t-T+1) f^{l}(u)+(t-T+1) g^{l}:=h_{T}^{l}(t), \\
& v^{l}(T-1)=0 .
\end{aligned}
$$

It is proved in [9, Theorem 3.10.1] that there is a positive Hölder constant $\beta>0$ such that

$$
\begin{aligned}
\left\|v^{l}\right\|_{C^{\beta, 2 \beta}([T, T+1] \times \Omega)} \leqslant & C\left\|h_{T}^{l}\right\|_{L^{\infty}\left([T-1, T+1], L^{2}(\Omega)\right)} \\
\leqslant & C\|g\|_{0,2}+C\|u\|_{L^{\infty}\left([T-1, T+1], L^{2}(\Omega)\right)} \\
& +C\|f(u)\|_{L^{\infty}\left([T-1, T+1], L^{2}(\Omega)\right)}
\end{aligned}
$$

Moreover, the constants $\beta>0$ and $C$ are independent of $\varepsilon$.

Inserting estimate (1.14) and (1.16) into the right-hand side of (1.27), we derive the following estimate:

$$
\begin{aligned}
\|u\|_{C^{\beta, 2 \beta}([T, T+1] \times \Omega)} & \leqslant C_{1}\|v\|_{C^{\beta, 2 \beta}([T, T+1] \times \Omega)} \\
& \leqslant Q\left(\left\|u_{0}\right\|_{0, \infty}\right) \mathrm{e}^{-\alpha T}+Q\left(\|g\|_{0,2}\right)
\end{aligned}
$$

which holds for $T \geqslant 1$. Estimates (1.21) and (1.22) are immediate corollaries of (1.25) and (1.28). Corollary 1.4 is proved.

We now derive some estimates for the time derivative $\partial_{t} u(t)$ for the solutions of $(0.1)$. To this end, we differentiate Eq. (0.1) with respect to $t$ and denote $\theta(t)=\partial_{t} u(t)$. Then, we obtain the equation

$$
\partial_{t} \theta(t)=A_{\varepsilon} \theta(t)-f^{\prime}(u(t)) \theta(t)
$$

Recall that $f \in C^{1}$, consequently (1.21) implies that

$$
\left\|f^{\prime}(u(t))\right\|_{0, \infty} \leqslant Q_{1}\left(\left\|u_{0}\right\|_{0, \infty}\right) \mathrm{e}^{-\alpha t}+Q_{1}\left(\|g\|_{0,2}\right)
$$

and, therefore, Eq. (1.29) also can be treated as the linear one. 
COROLlary 1.5. - Let $t \geqslant 1$. Then

$$
\left\|\partial_{t} u(t)\right\|_{0,2} \leqslant Q\left(\left\|u_{0}\right\|_{0, \infty}\right) \mathrm{e}^{-\alpha t}+Q\left(\|g\|_{0,2}\right),
$$

where the constant $\alpha>0$ and the function $Q$ is independent of $\varepsilon$.

Proof. - According to the smoothing property (1.20), we have

$$
\int_{T}^{T+1}\|\theta(t)\|_{0,2}^{2} \mathrm{~d} t \leqslant Q\left(\left\|u_{0}\right\|_{0, \infty}\right) \mathrm{e}^{-\alpha T}+Q\left(\|g\|_{0,2}\right),
$$

for $T \geqslant 1 / 2$. Taking the inner product in $\mathbb{R}^{k}$ of Eq. (1.29) with $(t-1 / 2) \theta(t)$ and integrating over $x \in \Omega$, we obtain using the integration by parts, and the uniform ellipticity assumption (0.4), that

$$
\begin{aligned}
& \partial_{t}\left((t-1 / 2)\|\theta(t)\|_{0,2}^{2}\right)+v\left((t-1 / 2)\|\theta(t)\|_{0,2}^{2}\right) \\
& \quad \leqslant C\|\theta(t)\|_{0,2}^{2}+C_{1}(t-1 / 2) \| f^{\prime}\left(u(t)\left\|_{0, \infty}\right\| \theta(t) \|_{0,2}^{2} .\right.
\end{aligned}
$$

Applying now the Gronwall inequality (starting with the time moment $t=1 / 2$ ) to estimate (1.33) and estimating the right-hand side of it by (1.32) and (1.30), we derive after the standard computatins the following estimate for $t \geqslant 1 / 2$ :

$$
\|\theta(t)\|_{0,2}^{2} \leqslant \frac{t+1}{t-1 / 2}\left(Q\left(\left\|u_{0}\right\|_{0, \infty}\right) \mathrm{e}^{-\alpha t}+Q\left(\|g\|_{0,2}\right)\right) .
$$

Restricting in (1.34) $t \geqslant 1$, we obtain estimate (1.31). Corollary 1.5 is proved.

Having estimate (1.31) for the time derivative $\theta=\partial_{t} u$ of the solutions of (1.29) and arguing as in the proof of the estimate (1.22), we can proof the following result.

COROLLARY 1.6. - Let the above assumptions hold and let $u(t)$ be a solution of equation (0.1). Then, there is the exponent $\beta>0$ such that $\partial_{t} u \in C^{\beta, 2 \beta}([T, T+1] \times \Omega)$, for $T \geqslant 2$, and the following estimate is valid:

$$
\left\|\partial_{t} u\right\|_{C^{\beta, 2 \beta}([T, T+1] \times \Omega)} \leqslant Q\left(\left\|u_{0}\right\|_{0, \infty}\right) \mathrm{e}^{-\alpha T}+Q\left(\|g\|_{0,2}\right), \quad T \geqslant 2,
$$

where the exponents $\beta, \alpha>0$ and the function $Q$ are independent of $\varepsilon$.

We summarize the obtained results in the following theorem.

THEOREM 1.2. - Let assumptions (0.2)-(0.6) hold. Then, for every fixed $\varepsilon>0$, problem (0.1) possesses a unique (in the class (0.7)) solution $u(t)$ and the following uniform (with respect to $\varepsilon$ ) estimate is valid:

$$
\|u(T)\|_{0, \infty}^{2}+\int_{T}^{T+1}\|u(t)\|_{1,2}^{2} \mathrm{~d} t \leqslant Q\left(\left\|u_{0}\right\|_{0, \infty}\right) \mathrm{e}^{-\alpha T}+Q\left(\|g\|_{0,2}\right),
$$

where $\alpha>0$ and $Q$ are independent of $\varepsilon$. 
Moreover, the following smoothing property is valid: if $T \geqslant 2$ then, the function $u \in C^{1+\beta, 2 \beta}([T, T+1] \times \Omega)$ and

$$
\begin{aligned}
& \|u(T)\|_{1,2}^{2}+\|u\|_{C^{1+\beta, 2 \beta}([T, T+1] \times \Omega)}^{2}+\int_{T}^{T+1}\left\|A_{\varepsilon} u(t)\right\|_{0,2}^{2} \mathrm{~d} t \\
& \leqslant Q\left(\left\|u_{0}\right\|_{0, \infty}\right) \mathrm{e}^{-\alpha T}+Q\left(\|g\|_{0,2}\right),
\end{aligned}
$$

where the exponents $\alpha, \beta>0$ and the function $Q$ are independent of $\varepsilon$.

Proof. - Indeed, estimates (1.36) and (1.37) has been obtained in Corollaries 1.1-1.6. The uniqueness of a solution in the class (0.7) is evident. The existence of a solution can be deduced from a priori estimates (1.36) and (1.37) by standard arguments (e.g. for the smooth initial data $u_{0}$ and smooth coefficients $a_{i j}^{l}$ the existence of a solution can be obtained by Leray-Schauder principle as in [9], the existence of a solution $u$ in general situation can be obtained approximating the initial data $u^{0}$ and the coefficients $a_{i j}^{l}$ by smooth ones $u_{0}^{n}$ and $a_{i j}^{l,(n)}$ and then passing to the limit $\left.n \rightarrow \infty\right)$. Theorem 1.2 is proved.

Remark 1.2. - As it has been already mentioned in Remark 1.1, the result of the theorem remains valid if the condition $(0.5)(2)$ is replaced by (1.15).

\section{The attractors}

In the previous section, we have proved that, for every fixed $\varepsilon>0$ and every $u_{0} \in$ $\Phi:=L^{\infty}(\Omega)$, problem $(0.1)$ possesses a unique solution $u(t) \in \Phi$ and, consequently, the semigroup

$$
S_{t}^{\varepsilon}: \Phi \rightarrow \Phi, \quad S_{t}^{\varepsilon} u_{0}:=u(t),
$$

where $u(t)$ is a solution of $(0.1)$, is correctly defined.

In this section, we prove that, for every $\varepsilon>0$, semigroup (2.1) generated by equation $(0.1)$ possesses an attractor $\mathcal{A}^{\varepsilon}$ in the phase space $\Phi$ and obtain several uniform (with respect to $\varepsilon>0$ ) estimates for these attractors which will be used in the next sections in order to study the homogenization limit $\varepsilon \rightarrow 0$. Moreover, in order to clarify the anisotropic dissipativity assumption (0.6), we give a number of examples of the righthand sides $f$ which admit the above theory.

For the convenience of the reader, we recall shortly the definition of the attractor and its basic properties (see e.g. $[1,6,13]$ for the detailed exposition).

DEFINITION 2.1. - Let $S_{t}^{\varepsilon}: \Phi \rightarrow \Phi$ be a semigroup, acting in a B-space $\Phi$. Then, a set $\mathcal{A}^{\varepsilon}$ is defined to be the attractor for $S_{t}^{\varepsilon}$ if the following is true:

(1) The set $\mathcal{A}^{\varepsilon}$ is compact in $\Phi$.

(2) The set $\mathcal{A}^{\varepsilon}$ is strictly invariant, i.e. $S_{t}^{\varepsilon} \mathcal{A}^{\varepsilon}=\mathcal{A}^{\varepsilon}$.

(3) The set $\mathcal{A}^{\varepsilon}$ is an attracting set for the semigroup $S_{t}^{\varepsilon}$, i.e. for every open neighbourhood $\mathcal{O}\left(\mathcal{A}^{\varepsilon}\right)$ of the set $\mathcal{A}^{\varepsilon}$ in $\Phi$ and for every bounded subset $B \subset \Phi$, there is $T=T(\mathcal{O}, B)$ such that

$$
S_{t}^{\varepsilon} B \subset \mathcal{O}\left(\mathcal{A}^{\varepsilon}\right), \quad \text { if } t \geqslant T \text {. }
$$


Remark 2.1. - The third condition of the previous definition is equivalent to the following: for every bounded subset $B \subset \Phi$

$$
\lim _{t \rightarrow \infty} \operatorname{dist}_{\Phi}\left(S_{t}^{\varepsilon} B, \mathcal{A}^{\varepsilon}\right)=0
$$

where dist ${ }_{\Phi}$ means the nonsymmetric Hausdorff distance, i.e.

$$
\operatorname{dist}_{\Phi}(V, W):=\sup _{v \in V} \inf _{w \in W}\|v-w\|_{\Phi} .
$$

It is also known (see e.g. [1]) that, if the attractor $\mathcal{A}^{\varepsilon}$ exists then it is generated by all complete bounded trajectories of the semigroup $S_{t}^{\varepsilon}$, i.e.

$$
\mathcal{A}^{\varepsilon}=\left\{u_{0} \in \Phi: \exists u \in L^{\infty}(R, \Phi), u(0)=u_{0} ; S_{t}^{\varepsilon} u(s)=u(t+s), \forall s \in \mathbb{R}, t \in \mathbb{R}_{+}\right\} .
$$

The following proposition gives the sufficient conditions for the existence of the attractor.

Proposition 2.1 [1]. - Let $S_{t}^{\varepsilon}: \Phi \rightarrow \Phi$ be an abstract semigroup in the space $\Phi$ which satisfies the following conditions:

(1) The semigroup $S_{t}^{\varepsilon}$ possesses a compact attracting set $K$ in $\Phi$ (in the sence of punkt (3)) of Definition 2.1).

(2) The operators $S_{t}^{\varepsilon}: \Phi \rightarrow \Phi$ have closed grafs, for every fixed $t \geqslant 0$ (as usual, it means that the set $\left\{\left(u_{0}, S_{t}^{\varepsilon} u_{0}\right): u_{0} \in \Phi\right\}$ is closed in $\left.\Phi \times \Phi\right)$.

Then, it possesses an attractor in $\mathcal{A}^{\varepsilon}$ in $\Phi$ and $\mathcal{A}^{\varepsilon} \subset K$.

We are now in position to state the main result of this section.

THEOREM 2.1. - Let the assumptions of Theorem 1.2 hold. Then, for every $\varepsilon>0$, semigroup (2.1) generated by equation (0.1) possesses an attractor $\mathcal{A}^{\varepsilon}$ in the phase space $\Phi:=L^{\infty}(\Omega)$ which admits the following description:

$$
\mathcal{A}^{\varepsilon}=\left.\mathcal{K}^{\varepsilon}\right|_{t=0},
$$

where $\mathcal{K}^{\varepsilon}$ is a collection of all bounded (with respect to $t \in \mathbb{R}$ ) solutions $\hat{u}(t)$ of the equation

$$
\partial_{t} \hat{u}(t)=A_{\varepsilon} \hat{u}(t)-f(\hat{u}(t))+g, \quad t \in \mathbb{R},\left.\quad \hat{u}\right|_{\partial \Omega}=0 .
$$

Proof. - Let us verify the assumptions of Proposition 2.1. Indeed, it follows from estimate (1.37) that the ball $K_{R}$ of radius $R$ in the space $\Phi_{\beta}:=C^{2 \beta}(\Omega)$ is an attracting set for semigroup (2.1) if $R$ is large enough (namely if $R>Q\left(\|g\|_{0,2}\right)$ ). Since the embedding $\Phi_{\beta} \subset \Phi$ is compact then the set $K_{R}$ is the (pre)compact attracting set for semigroup (2.1). Taking its closure in $\Phi$, we construct the compact attracting set for $S_{t}^{\varepsilon}$. Thus, the first assumption of Proposition 2.1 is verified.

Let us verify the second one. Recall, that we should prove that if $u_{0}^{n} \rightarrow u_{0}$ in $\Phi$ and $u_{n}(T):=S_{T}^{\varepsilon} u_{0} \rightarrow v$ in $\Phi$ then $v=u(T):=S_{T}^{\varepsilon} u_{0}$.

We first note that the operator $S_{T}^{\varepsilon}$ is uniformly Liptshitz continuous with respect to the $L^{2}$-norm on every bounded subset $B$ in $\Phi$. Indeed, let $u_{0}^{1}, u_{0}^{2} \in B$ be an arbitrary initial 
data, $u_{i}(t)$ be the corresponding solutions of problem $(0.1)$ and $w(t)=u_{1}(t)-u_{2}(t)$. Then, this function satisfies the equation

$$
\partial_{t} w=A_{\varepsilon} w-l(t) w,\left.\quad w\right|_{t=0}=u_{0}^{1}-u_{2}^{0},\left.\quad w\right|_{\partial \Omega}=0,
$$

where $l(t):=\int_{0}^{1} f^{\prime}\left(s u_{1}(t)+(1-s) u_{2}(t)\right) \mathrm{d} s$. It follows from estimate (1.21) that $\left\|u_{i}(t)\right\|_{0, \infty} \leqslant C_{B}$ where the constant $C_{B}$ depends only on the norm $\|B\|_{\Phi}$ and, consequently

$$
\|l(t)\|_{0, \infty} \leqslant C_{1}(B) .
$$

Taking the inner product in $\mathbb{R}^{k}$ with the function $w(t)$, integrating over $x \in \Omega$, estimating $l(t)$ by (2.9) and applying the Gronwall inequality, we derive the estimate

$$
\left\|u_{1}(T)-u_{2}(T)\right\|_{0,2}^{2} \leqslant C \mathrm{e}^{K T}\left\|u_{1}(0)-u_{2}(0)\right\|_{0,2}^{2},
$$

where the constants $C$ and $K$ depend only on $\|B\|_{\Phi}$.

Having $L^{2}$-Lipschitz continuity (2.10), we immediately conclude that $S_{T}^{\varepsilon}$ has a closed graph in $\Phi$. Indeed, let $u_{0}^{n} \rightarrow u_{0}$ in $\Phi$ and $u_{n}(T) \rightarrow v$ in $\Phi$. Then, according to (2.10) $u_{n}(T) \rightarrow u(T)$ in $L^{2}(\Omega)$ and, consequently, $v=u(T)$.

Thus, all assumptions of Proposition 2.1 are verified for semigroup (2.1) generated by Eq. (0.1) and, therefore, this semigroup possesses an attractor $\mathcal{A}^{\varepsilon}$. Description (2.6) is an immediate corollary of the formula (2.5). Theorem 2.1 is proved.

The following corollary is of fundamental significance for our study the homogenization $\operatorname{limit}\left(\lim _{\varepsilon \rightarrow 0} \mathcal{A}^{\varepsilon}\right)$ in the next sections.

COROLlaRY 2.1. - The attractors $\mathcal{A}^{\varepsilon} \in \Phi_{\beta} \cap W_{0}^{1,2}(\Omega)$ and uniformly bounded with respect to $\varepsilon>0$ in this space:

$$
\left\|\mathcal{A}^{\varepsilon}\right\|_{1,2}+\left\|\mathcal{A}^{\varepsilon}\right\|_{C^{2 \beta}(\Omega)} \leqslant Q\left(\|g\|_{0,2}\right) .
$$

Here the exponent $\beta>0$ and the function $Q$ are independent of $\varepsilon$.

Moreover, let $\hat{u}(t) \in \mathcal{K}^{\varepsilon}$ be a bounded solution of Eq. (2.7). Then

$$
\|\hat{u}\|_{C^{1+\beta, 2 \beta}([T, T+1] \times \Omega)}^{2}+\|\hat{u}(T)\|_{1,2}^{2}+\int_{T}^{T+1}\left\|A_{\varepsilon} \hat{u}(t)\right\|_{0,2}^{2} \mathrm{~d} t \leqslant Q\left(\|g\|_{0,2}\right),
$$

where the function $Q$ is independent of $\varepsilon, \hat{u}$, and $T \in \mathbb{R}$.

Indeed, the first estimate is an immediate corollary of the second one together with representation (2.6) and estimate (2.12) can be easily derived from estimate (1.37) and from the fact that $\|\hat{u}(t)\|_{0, \infty}$ remains bounded when $t \rightarrow-\infty$.

Recall that we have proved the attractor's existence Theorem 2.1 under anisotropic dissipativity assumption (0.6) on the nonlinear term $f$ which looks a little unusual. In conclusion of this section, we discuss this assumption and give a number of examples where it is satisfied. 
We first note that, in a scalar case $k=1$, we need only the following natural assumption on the nonlinear term $f$ : there is $R_{f}<\infty$ such that

$$
\operatorname{sgn} v f(v) \geqslant 0 \quad \text { if }|v|>R_{f}
$$

(which as known is essential in order to obtain the global existence of solutions of (0.1)). Note also that, in this case, the polynomial growth restriction $(0.5)(2)$ is also can be removed due to the maximum principle.

Consider now the case of systems $(k \geqslant 2)$. The usual (for the attractor's industry) assumptions for the nonlinearity $f$ are the following:

$$
\left\{\begin{array}{l}
\text { 1. } f(u) \cdot u \geqslant-C+C_{1}|u|^{p+1}, \\
\text { 2. }|f(u)| \leqslant C\left(1+|u|^{p}\right), \quad p<p_{c}, \\
\text { 3. }\left|f^{\prime}(u)\right| \leqslant C\left(1+|u|^{p-1}\right)
\end{array}\right.
$$

(see e.g. [1,13]) which involve the growth restrictions $p<p_{c}$. Unfortunately, the limit exponent $p_{c}$ in assumptions (2.14) is too restrictive $\left(p_{c}=1+4 / n=7 / 3<3\right.$ (see [1])) and, consequently, even the cubic nonlinearities are out of the consideration).

Another standard possibility is to impose the additional quasi-monotonicity assumption

$$
f^{\prime}(u) \geqslant-K
$$

to the nonlinear term $f$. In this case, the global existence and uniqueness of weak solutions and even the existence of the attractors $\mathcal{A}^{\varepsilon}$ in $L^{2}(\Omega)$ can be obtained without the growth restriction $p<p_{c}$ (analogously to [1]). Moreover, in the case where $A_{\varepsilon}=A=a \Delta_{x}, a \in \mathcal{L}\left(\mathbb{R}^{k}, \mathbb{R}^{k}\right)$ with $a+a^{*}>0$, the considerable theory (which includes the $L^{\infty}$-bounds for weak solutions, their smoothness, the differentiability of the corresponding semigroup, the finite dimensionality of the attractors, etc.) can be constructed for Eq. (0.1) with essentially weaker growth restriction $p<1+4 /(n-4)$. For instance, if $n=3$ and $A_{\varepsilon}:=a \Delta_{x}$, the following assumptions on $f$

$$
\begin{array}{ll}
\text { 1. } f \in C^{1}\left(\mathbb{R}^{k}, \mathbb{R}^{k}\right), \quad \text { 2. } f(u) \cdot u \geqslant-C, \quad \text { 3. } f^{\prime}(u) \geqslant-K
\end{array}
$$

are sufficient (see [18]). But the proof of these results is essentially based on the trick with multiplication of Eq. (0.1) by $\Delta_{x} u$ and does not work in our situation where the operator $A_{\varepsilon}$ has the form (0.2), (0.3). Therefore, even under the additional assumption (2.15), we do not know how to obtain the additional regularity of weak solutions of $(0.1)$ (which is necessary to study the limit behaviour as $\varepsilon \rightarrow 0$ of attractors $\mathcal{A}^{\varepsilon}$ ) without the growth restriction $p<p_{c}<7 / 3$.

In order to remove this extremely restrictive growth condition, we suggest to use the dissipativity assumption in a new form (0.6) which, on the one hand, is not very restrictive (as the examples given below show) and, on the other hand, admits to obtain the $L^{\infty}$-bounds of solutions of $(0.1)$ (with the diagonal leading part $A_{\varepsilon}$, see $(0.2)$ ) without the growth restrictions. We illustrate this anisotropic dissipativity assumption by several examples. 
Assume, for the first, that the nonlinearity $f$ possesses the following decomposition:

$$
f(v)=f_{1}(v)+f_{2}(v), \quad \text { where } f_{1}(v):=\operatorname{diag}\left\{f_{1}^{1}\left(v_{1}\right), \ldots, f_{1}^{k}\left(v_{k}\right)\right\}
$$

and the functions $f_{1}^{i}\left(v^{i}\right)$ satisfy the assumptions

$$
f_{1}^{i}\left(v^{i}\right) \cdot v^{i} \geqslant-C_{i}+\alpha_{i}\left|v^{i}\right|^{q_{i}+2}, \quad i=1, \ldots, k,
$$

for the appropriate $q_{i} \geqslant 0, \alpha_{i}>0$ and the functions $f_{2}(v)$ satisfy the following growth restrictions:

$$
\left|f_{2}^{i}(v)\right| \leqslant C\left(1+|v|^{l_{i}}\right), \quad i=1, \ldots, k, l_{i} \geqslant 0 .
$$

Assume also that

$$
l_{i}<1+q_{i}, \quad i=1, \ldots, k .
$$

Lemma 2.1. - Let assumptions (2.17)-(2.20) hold. Then, for every $q \geqslant q_{i}, i=$ $1, \ldots, k$, nonlinearity (2.17) satisfies the anisotropic dissipativity condition (0.6) with the exponents $p_{i}=q-q_{i}$.

Proof. - Indeed, due to (2.18)

$$
\sum_{i=1}^{k} f_{1}^{i}\left(v^{i}\right) \cdot v^{i}\left|v^{i}\right|^{p_{i}} \geqslant-C+1 / 2 \sum_{i=1}^{k} \alpha_{i}\left|v^{i}\right|^{q+2} \geqslant-C+\beta|v|^{q+2} .
$$

It follows from restrictions (2.19) and (2.20) and Hölder inequality that

$$
\begin{aligned}
\left|f_{2}^{i}(v)\right| \cdot\left|v^{i}\right| \cdot\left|v^{i}\right|^{q-q_{i}} & \leqslant \mu\left|v^{i}\right|^{q+2}+C_{\mu}\left|f_{2}^{i}(v)\right|^{(q+2) /\left(q_{i}+1\right)} \\
& \leqslant \mu|v|^{q+2}+C_{\mu}+C_{\mu}|v|^{(q+2) l_{i} /\left(q_{i}+1\right)} \\
& \leqslant 2 \mu|v|^{q+2}+C_{\mu}^{\prime},
\end{aligned}
$$

where $\mu>0$ is an arbitrary positive number. Estimates (2.21) and (2.22) prove the lemma.

Remark 2.2. - Note that, under the assumptions of Lemma 2.1, (0.6) is valid with $p_{i}=q-q_{i}$ where $q$ may be arbitrary large. Consequently, for every nonlinearity (2.17) with the polynomial rate of growth (i.e., for which $(0.5)(2)$ is satisfied), we may satisfy also the assumption $p_{i} \geqslant 2(p-1)$ and, therefore, Theorem 2.1 is valid for such nonlinearities.

Example 2.1. - The simplest example of such nonlinearities is the following:

$$
f_{1}(v)=\operatorname{diag}\left\{\alpha_{1} v^{1}\left|v^{1}\right|^{q_{1}}, \ldots, \alpha_{k} v^{k}\left|v^{k}\right|^{q_{k}}\right\},
$$

with $q_{i}, \alpha_{i}>0$ and $f_{2}$ is linear $f_{2}(v)=L v$. Then, all assumptions of Lemma 2.1 are, obviously, satisfied and, consequently, Theorem 2.1 holds.

Example 2.2. - Consider the case $k=2, v=\left(v_{1}, v_{2}\right)$ and the nonlinearity

$$
f(v)=\left(\begin{array}{c}
v_{1}^{3}-\alpha v_{1}-\beta v_{2} \\
v_{2}-\gamma v_{1}
\end{array}\right), \quad \alpha, \beta, \gamma \in \mathbb{R}
$$


which corresponds to the Fitz-Nagumo system (see [8]). We note that the assumptions of Lemma 2.1 are violated $\left(q_{2}=0, l_{2}=1, l_{2}=q_{2}+1\right)$ here. Nevertheless, a simple computations show that the dissipativity assumption (0.6) is valid with the exponents $p_{1}=p_{2}=q$ and every $q \geqslant 0$, consequently, Theorem 2.1 remains true for nonlinearity (2.24).

Example 2.3. - Consider the case $k=2$ and the nonlinearity

$$
f(v)=\left(\begin{array}{c}
v_{1}^{3}-\alpha v_{2} \\
v_{2}-\beta v_{1}^{3}
\end{array}\right), \quad 2|\alpha|+|\beta| \leqslant 3,2|\beta|+|\alpha| \leqslant 3 .
$$

If $\alpha=\beta=1$ then (2.25) satisfies the equation $f^{1}(v)+f^{2}(v)=0$ which is natural from the reaciton-diffusion point of view. Note that this nonlinearity evidently does not satisfy the standard dissipativity assumption $(f(v) . v \geqslant-C)$. Nevertheless, the elementary computation shows that the anisotropic dissipativity assumption is satisfied with $p_{1}=5$ and $p_{2}=1$. Note also that function (2.24) satisfies also condition (1.15) with these exponents. Consequently, Theorem 2.1 holds for this nonlinearity.

Example 2.4. - We conclude the section by the following 'exotic' example:

$$
f(v)=\left(\begin{array}{l}
v_{1}^{3}-v_{2} \\
v_{2}^{3}-v_{1}^{5}
\end{array}\right)
$$

of two RDEs coupled by the monom $v_{1}^{5}$ of the highest order. Nevertheless, the anisotropic dissipativity condition is valid with the exponents $p_{2}=4$ and $p_{1}=10$. Since (1.15) is also hold for this exponents then Theorem 2.1 is valid for nonlinearity (2.26).

\section{The homogenization}

This section is devoted to study the behavior of the attractors $\mathcal{A}^{\varepsilon}$ constructed in the previous section when $\varepsilon \rightarrow 0$. The main task of the section is to prove that these attractors tend as $\varepsilon \rightarrow 0$ to the attractor $\mathcal{A}^{0}$ of the homogenized problem (0.9). In order to write this homogenized system, we recall briefly some known results from the theory of almostperiodic homogenization (see e.g. [20] for the detailed exposition).

Recall, that every almost-periodic function $w \in A P\left(\mathbb{R}^{3}\right)$ possesses the mean value which can be calculated by the following formula:

$$
\langle w\rangle:=\lim _{T \rightarrow \infty} \frac{1}{2^{3} T^{3}} \int_{[-T, T]^{3}} w(x) \mathrm{d} x
$$

and the following Fourier expansion

$$
w(x)=\sum_{\hat{w}(\xi) \neq 0} \hat{w}(\xi) \mathrm{e}^{\mathrm{i}(x, \xi)},
$$

where the amplitudes $\hat{w}(\xi) \in \mathbb{C}, \xi \in \mathbb{R}^{3}$ can be found by

$$
\hat{w}(\xi):=\left\langle w(x) \mathrm{e}^{-\mathrm{i}(x, \xi)}\right\rangle
$$


(see e.g. $[10,11]$ ). It is known that the set $\sigma(w):=\left\{\xi \in \mathbb{R}^{3}, \hat{w}(\xi) \neq 0\right\}$ is not greater than countable, therefore, the sum (3.1) has a sense. Moreover,

$$
\sum_{\xi \in \sigma(w)}|\hat{w}(\xi)|^{2}<\infty
$$

and series (3.1) converge to the function $w$ in the sense of the Bezikovich norm $\|v\|_{B^{2}\left(\mathbb{R}^{3}\right)}^{2}:=\langle v(x) \bar{v}(x)\rangle$ (see [10] for details).

As usual, we denote by $\operatorname{Trig}^{\circ}\left(\mathbb{R}^{3}\right)$ the space of all finite trigonometric polynomials of the form (3.1)

$$
\operatorname{Trig}^{\circ}\left(\mathbb{R}^{3}\right):=\left\{w(x)=\sum_{k=1}^{N} w_{k} \mathrm{e}^{\mathrm{i}\left(x, \xi_{k}\right)}: N \in \mathbb{N}, \xi_{k} \in \mathbb{R}^{3}, w_{k} \in \mathbb{C}, k=1, \ldots, N\right\} .
$$

Now we are ready to write the formula for the homogenized operator $A_{0}$ for problem (0.1). To this end, we first define the functions $\mathcal{B}^{l}(\xi), \xi \in \mathbb{R}^{3}, l=1, \ldots, k$, by

$$
\mathcal{B}^{l}(\xi):=\inf _{N \in \operatorname{Trig}^{0}\left(\mathbb{R}^{3}\right)}\left\langle\sum_{i, j} a_{i j}^{l}(y)\left(\xi_{i}+\partial_{y_{i}} N(y)\right)\left(\xi_{j}+\partial_{y_{j}} N(y)\right)\right\rangle,
$$

where $a_{i j}^{l}(y)$ has been defined in (0.3). Note, that (3.5) has a sense since the expression inside of $\langle\cdot\rangle$ is, obviously, an almost-periodic function.

It is known (see [20]) that the functions $\mathcal{B}^{l}(\xi)$ generate positive definite quadratic forms with respect to $\xi$, i.e.

$$
\mathcal{B}^{l}(\xi)=\sum_{i, j} \tilde{a}_{i j}^{l} \xi_{i} \xi_{j}, \quad \tilde{a}_{i j}^{l} \in \mathbb{R} .
$$

Define now the operators $A_{0}^{l}$ and the operator $A_{0}$ in the following way:

$$
A_{0}^{l} u^{l}:=\sum_{i, j} \partial_{x_{j}}\left(\tilde{a}_{i j}^{l} \partial_{x_{i}} u^{l}\right), \quad A_{0} u:=\operatorname{diag}\left\{A_{0}^{1} u^{1}, \ldots, A_{0}^{k} u^{k}\right\} .
$$

This choice of the leading part of the homogenized equation for (0.1) is justified by the following proposition.

Proposition 3.1 [20]. - Let the functions $v^{\varepsilon} \in W_{0}^{1,2}(\Omega)$ be the solutions of the following problem:

$$
A_{\varepsilon}^{l} v^{\varepsilon}=h, \quad h \in W^{-1,2}(\Omega),
$$

where $l \in\{1, \ldots, k\}$ is fixed and the operator $A_{\varepsilon}^{l}$ is defined by (0.3). Then, $v^{\varepsilon} \rightarrow v^{0}$ weakly in $W_{0}^{1,2}(\Omega)$ as $\varepsilon \rightarrow 0$ and the function $v^{0} \in W_{0}^{1,2}(\Omega)$ is a solution of the limit problem

$$
A_{0}^{l} v^{0}=h,
$$

where the operator $A_{0}^{l}$ is defined by (3.5)-(3.7). 
Let us consider the homogenized equation

$$
\left\{\begin{array}{l}
\partial_{t} u=A_{0} u-f(u)+g \\
\left.u\right|_{t=0}=u_{0},\left.\quad u\right|_{\partial \Omega}=0
\end{array}\right.
$$

for the non-homogeneous equation (0.1). Note that this equation satisfies all assumptions of Theorem 2.1 (due to the fact that the forms $\mathcal{B}^{l}(\xi)$ are positive defined) and, consequently, possesses the attractor $\mathcal{A}^{0}$ in the phase space $\Phi=L^{\infty}(\Omega)$. Moreover, estimates (1.11) and (2.12) remain valid for the limit equation (3.10).

The main result of this section is the following theorem.

THEOREM 3.1. - Let the assumptions of Theorem 2.1 hold and let $\mathcal{A}^{0}$ be the attractor of the limit $(\varepsilon=0)$ problem (3.10). Then, the attractors $\mathcal{A}^{\varepsilon}$ of $(0.1)$ converge to $\mathcal{A}^{0}$ as $\varepsilon \rightarrow 0$ in the following sense:

$$
\lim _{\varepsilon \rightarrow 0} \operatorname{dist}_{C^{2 \beta^{\prime}}(\Omega)}\left(\mathcal{A}^{\varepsilon}, \mathcal{A}^{0}\right)=0,
$$

for the appropriate sufficiently small positive exponent $\beta^{\prime}>0$.

Proof. - As usual (see [1]), in order to prove upper-semicontinuity (3.11), we should consider an arbitrary sequence $\varepsilon_{n} \rightarrow 0$ and $u_{0, n} \in \mathcal{A}^{\varepsilon_{n}}$ and prove that it is possible to extract from it a subsequence $u_{0, n_{k}} \rightarrow u_{0} \in \mathcal{A}^{0}$ in $\Phi_{\beta^{\prime}}$.

Let us fix an arbitrary sequence $\varepsilon_{n} \rightarrow 0$ and an arbitrary sequence $u_{0, n} \in \mathcal{A}^{\varepsilon_{n}}$. Let $\hat{u}_{n}(t) \in \mathcal{K}^{\varepsilon_{n}}$ be the corresponding bounded solutions of Eq. (2.7) (with $\varepsilon$ replaced by $\varepsilon_{n}$ ) such that $u_{0, n}=\hat{u}_{n}(0)$ (which exist due to representation (2.6)). Then, according to $(2.12)$

$$
\left\|\hat{u}_{n}\right\|_{C^{1+\beta, 2 \beta}([T, T+1] \times \Omega)} \leqslant Q\left(\|g\|_{0,2}\right),
$$

with the appropriate $\beta>0$ and the function $Q$ independent of $T \in \mathbb{R}$ and $n \in \mathbb{N}$. Let us fix $0<\beta^{\prime}<\beta$. Then, due to the compactness of the embedding

$$
C^{1+\beta^{\prime}, 2 \beta^{\prime}}([T, T+1] \times \Omega) \Subset C^{1+\beta, 2 \beta}([T, T+1] \times \Omega),
$$

and due to Cantor diagonal procedure, we may assume (passing to a subsequence if necessary) that there is a function $\hat{u} \in C^{1+\beta^{\prime}, 2 \beta^{\prime}}([T, T+1] \times \Omega)$ such that

$$
\hat{u}_{n} \rightarrow \hat{u}, \quad \partial_{t} \hat{u}_{n} \rightarrow \partial_{t} \hat{u} \quad \text { as } n \rightarrow \infty \text { in the space } C^{\beta^{\prime}, 2 \beta^{\prime}}([T, T+1] \times \Omega),
$$

for every fixed $T \in \mathbb{R}$. Particularly, $\hat{u}_{n}(0) \rightarrow \hat{u}(0)$ in $\Phi_{\beta^{\prime}}$. Therefore, if $\hat{u} \in \mathcal{K}^{0}$ then, due to (2.6), $u_{0}=\hat{u}(0) \in \mathcal{A}^{0}$. Thus, there remains to prove that the limit function $\hat{u}(t)$ is a bounded solution of the limit equation (3.10). This fact can be easily verified using Proposition 3.1 and convergence (3.13). Indeed, let us verify that the function $u^{l}(t)$ satisfies the $l$ th equation of (3.10). Since $\hat{u}$ and $\partial_{t} \hat{u}$ are continuous with respect to $(t, x)$ it is sufficient to verify this identity for every fixed $T \in \mathbb{R}$. To this end, we rewrite the $l$ th equation of (1.2) in the form of elliptic boundary problem:

$$
A_{\varepsilon_{n}}^{l} \hat{u}_{n}^{l}(T)=h_{n}^{l}(T):=\partial_{t} \hat{u}_{n}^{l}(T)+f^{l}(\hat{u}(T))-g^{l},\left.\quad \hat{u}^{l}(T)\right|_{\partial \Omega}=0 .
$$


Note that convergence (3.13) implies that

$$
h_{n}^{l}(T) \rightarrow h_{0}^{l}(T):=\partial_{t} \hat{u}^{l}(T)+f^{l}(\hat{u}(T))-g^{l}
$$

in the space $C^{2 \beta_{1}}(\Omega)$.

Let $v_{n} \in W_{0}^{1,2}(\Omega)$ be a solution of the following elliptic boundary problem:

$$
A_{\varepsilon_{n}}^{l} v_{n}=h_{0}^{l}(T)
$$

Then, on the one hand

$$
\left\|\hat{u}_{n}^{l}(T)-v_{n}\right\|_{1,2} \leqslant C\left\|h_{n}^{l}(T)-h_{0}^{l}(T)\right\|_{-1,2} \rightarrow 0,
$$

due to (3.15) and due to the uniform with respect to $\varepsilon$ boundedness of $\left(A_{\varepsilon}\right)^{-1}: W^{-1,2}(\Omega)$ $\rightarrow W_{0}^{1,2}(\Omega)$. On the other hand, due to Proposition 3.1

$$
v^{n} \rightarrow v_{0}, \quad \text { in } W_{0}^{1,2}(\Omega) \text { where } A_{0}^{l} v_{0}=h_{0}^{l}(T) .
$$

Convergences (3.17) and (3.18) imply that $\hat{u}^{l}(T)=v_{0}$ and, consequently

$$
A_{0}^{l} \hat{u}^{l}(T)=h_{0}^{l}(T)=\partial_{t} u^{l}(T)+f^{l}(\hat{u}(T))-g^{l} .
$$

Since $T$ is arbitrary then the function $u^{l}(t)$ really satisfies the $l$ th equation of (3.10) and, therefore, (since $l \in\{1, \ldots, k\}$ is arbitrary) $\hat{u}$ satisfies the homogenized equation (3.10). Note also that the uniform estimate (3.12) and convergence (3.13) imply that $\hat{u}$ is bounded. Thus, $\hat{u} \in \mathcal{K}^{0}$. Theorem 3.1 is proved.

\section{The case of periodic coefficients: estimates of the error}

This section is devoted to a more detailed consideration of the particular case where the coefficients $a_{i j}^{l}(y)$ are periodic and smooth $\left(C^{2}\left(\mathbb{R}^{3}\right)\right)$ functions in $\mathbb{R}^{3}$, i.e it is assumed that there are positive numbers $\vec{T}=\left(T_{1}, T_{2}, T_{3}\right)>0$ such that

$$
a_{i j}^{l}(y+(\vec{T}, m)) \equiv a_{i j}^{l}(y), \quad \text { for all } i, j, l \text { and for all } m \in \mathbb{Z}^{3}, y \in \mathbb{R}^{3} .
$$

In this case, using the method of asymptotic expansions (see $[2,20]$ ), we obtain the error estimates for the approximation of the individual solutions of $(0.1)$ by the solutions of the homogenized problem (3.10) and based on these estimates we derive the estimates for the distance between the global attractors $\mathcal{A}^{\varepsilon}$ and $\mathcal{A}^{0}$ under some additional assumptions on the limit attractor $\mathcal{A}^{0}$.

THEOREM 4.1. - Let the assumptions of Theorem 3.1 hold and let in addition (4.1) be also valid. Then, for every $\varepsilon>0$ small enough and every $u_{0} \in \Phi \cap W_{0}^{1,2}(\Omega)$, the following estimate is valid:

$$
\left\|u_{\varepsilon}(t)-\hat{u}(t)\right\|_{0,2} \leqslant Q\left(\left\|u_{0}\right\|_{L^{\infty} \cap W_{0}^{1,2}}\right) \varepsilon^{1 / 3} \mathrm{e}^{K t},
$$


where $u_{\varepsilon}(t)=S_{t}^{\varepsilon} u_{0}, \hat{u}(t)=S_{t}^{0} u_{0}$ are the solutions of problems (0.1) and (3.10) respectively, the function $Q$ and the constant $K=K\left(\left\|u_{0}\right\|_{0, \infty}\right)$ are independent of $\varepsilon$.

Proof. - We first note that, since the elliptic operator $A_{0}$ has constant coefficients, we can derive from (1.17) that

$$
\begin{gathered}
\|\hat{u}(T)\|_{1,2}^{2}+\int_{T}^{T+1}\|\hat{u}(t)\|_{2,2}^{2} \mathrm{~d} t+\int_{T}^{T+1}\left\|\partial_{t} \hat{u}(t)\right\|_{0,2}^{2} \mathrm{~d} t \\
\leqslant Q\left(\left\|u_{0}\right\|_{0, \infty}+\left\|u_{0}\right\|_{1,2}\right)+Q\left(\|g\|_{0,2}\right),
\end{gathered}
$$

for the appropriate function $Q$ independent of $T \geqslant 0$ (here we have implicitly used the elliptic regularity estimate $\|u\|_{2,2} \leqslant C\left\|A_{0} u\right\|_{0,2}$ ).

Let us fix $l \in\{1, \ldots, k\}$, consider the $l$ th equation of $(0.1)$ :

$$
\partial_{t} u_{\varepsilon}^{l}=A_{\varepsilon}^{l} u_{\varepsilon}^{l}-f^{l}\left(u_{\varepsilon}\right)+g^{l} ;\left.\quad u_{\varepsilon}^{l}\right|_{t=0}=u_{0}^{l}
$$

and introduce the correctors $N_{k}^{l}(y), k=1,2,3$, as solutions of the following auxiliary periodic problems:

$$
\left\{\begin{array}{l}
\sum_{i, j=1}^{3} \partial_{y_{i}}\left(a_{i j}^{l}(y) \partial_{y_{j}} N_{k}^{l}(y)\right)=-\sum_{i=1}^{3} \partial_{y_{i}} a_{i k}^{l}(y), \quad y \in \mathbb{R}^{3}, \\
N_{k}^{l}(y+(\vec{T}, m)) \equiv N_{k}^{l}(y), \quad m \in \mathbb{Z}^{3} .
\end{array}\right.
$$

It is well-known that the periodic problems (4.5) have unique solutions (due to the uniform ellipticity assumption (0.4)) and since $a_{i j}^{l}(y)$ are smooth $\left(C^{2}\right)$ then

$$
\left\|N_{k}^{l}\right\|_{C_{b}^{1}\left(\mathbb{R}^{3}\right)} \leqslant C
$$

Moreover, if the solutions of (4.5) are known then the coefficients $\tilde{a}_{i j}^{l}$ of the limit elliptic operator $A_{0}^{l}$ can be calculated by the following formulae (see e.g. [20]):

$$
\tilde{a}_{i j}^{l}=\left\langle a_{i j}^{l}(y)\right\rangle+\sum_{k=1}^{3}\left\langle a_{i k}^{l}(y) \partial_{y_{k}} N_{j}(y)\right\rangle .
$$

Lemma 4.1. - Define the functions

$$
\tilde{u}_{\varepsilon}^{l}(t):=\hat{u}^{l}(t)+\varepsilon \sum_{k=1}^{3} N_{k}^{l}\left(\varepsilon^{-1} x\right) \partial_{x_{k}} \hat{u}^{l}(t) .
$$

Then

$$
\left\|A_{\varepsilon}^{l} \tilde{u}_{\varepsilon}^{l}(t)-A_{0}^{l} \hat{u}^{l}(t)\right\|_{-1,2} \leqslant C \varepsilon\left\|\hat{u}^{l}(t)\right\|_{2,2},
$$

where the constant $C$ is independent of $\varepsilon$.

Proof. - Indeed, it is computed in [20, p. 27] that

$$
\sum_{j=1}^{3}\left(a_{i j}^{l}\left(\varepsilon^{-1} x\right) \partial_{x_{j}} \tilde{u}_{\varepsilon}^{l}-\tilde{a}_{i j}^{l} \partial_{x_{j}} \hat{u}^{l}\right)=\varepsilon \sum_{j=1}^{3} \partial_{x_{j}}\left(\alpha_{i j}^{l k}\left(\varepsilon^{-1} x\right) \partial_{x_{k}} \hat{u}^{l}\right)+r_{\varepsilon}^{i l},
$$


where $\alpha_{i j}^{l k}(y)$ are certain periodic functions such that

$$
\alpha_{i j}^{l k}(y)=-\alpha_{j i}^{l k}(y)
$$

and the remainders $r_{\varepsilon}^{i l}$ are

$$
r_{\varepsilon}^{i l}:=\varepsilon \sum_{k, j=1}^{3} N_{k}^{l}\left(\varepsilon^{-1} x\right) \partial_{x_{j} x_{k}}^{2} \hat{u}^{l}-\varepsilon \alpha_{i j}^{k l}\left(\varepsilon^{-1} x\right) \partial_{x_{j} x_{k}}^{2} \hat{u}^{l} .
$$

Note that due to (4.11) the divergence from the first term into the right-hand side of (4.10) equals zero and, consequently

$$
\left\|A_{\varepsilon}^{l} \tilde{u}_{\varepsilon}^{l}(t)-A_{0}^{l} \hat{u}^{l}(t)\right\|_{-1,2} \leqslant \sum_{i=1}^{3}\left\|r_{\varepsilon}^{i l}(t)\right\|_{0,2} \leqslant C \varepsilon\left\|\hat{u}^{l}(t)\right\|_{2,2} .
$$

Lemma 4.1 is proved.

Note that the constructed functions $\tilde{u}_{\varepsilon}^{l}(t)$ do not satisfy the boundary conditions $\left(\tilde{u}_{\varepsilon}^{l}(t) \notin W_{0}^{1,2}(\Omega)\right)$ which is inconvenient for our purposes (we are planning to multiply equation $(4.4)$ in $L^{2}(\Omega)$ by $\tilde{u}_{\varepsilon}^{l}(t)-u_{\varepsilon}^{l}(t)$ and integrate by parts). In order to avoid this difficulty, we introduce (following to [20]) the family $\tau_{\varepsilon}(x) \in C_{0}^{\infty}(\Omega)$ of cut-off functions satisfying the following conditions:

(1) $0 \leqslant \tau_{\varepsilon} \leqslant 1$, and $\tau_{\varepsilon}(x) \equiv 1$ if $x \in \Omega \backslash \mathcal{O}_{\varepsilon}(\partial \Omega)$, where $\mathcal{O}_{\varepsilon}(\partial \Omega)$ means the $\varepsilon$-neighbourhood of the boundary $\partial \Omega$.

(2) $\varepsilon\left|\nabla_{x} \tau_{\varepsilon}(x)\right| \leqslant C$ for every $x \in \Omega$ and $\varepsilon>0$.

(Such family exists because the boundary $\partial \Omega$ is assumed to be smooth (see [20])) and make the following boundary correction of the functions $\tilde{u}_{\varepsilon}^{l}(t)$ :

$$
w_{\varepsilon}^{l}(t):=\tilde{u}_{\varepsilon}^{l}(t)-\varepsilon\left(1-\tau_{\varepsilon}(x)\right) \sum_{k=1}^{3} N_{k}\left(\varepsilon^{-1} x\right) \partial_{x_{k}} \hat{u}^{l}(t) .
$$

Then, obviously, $w_{\varepsilon}^{l}(t) \in W_{0}^{1,2}(\Omega)$ (to be more precise $w_{\varepsilon}^{l} \in L^{2}\left([0, T], W_{0}^{1,2}(\Omega)\right)$ ) and the $W^{1,2}$-distance between $\tilde{u}_{\varepsilon}^{l}$ and $w_{\varepsilon}^{l}$ is sufficiently small as the following lemma shows.

LEMMA 4.2. - Let the functions $\tilde{u}_{\varepsilon}^{l}(t)$ and $w_{\varepsilon}^{l}(t)$ be defined by (4.8) and (4.13) respectively. Then

$$
\left\|\tilde{u}_{\varepsilon}^{l}-w_{\varepsilon}^{l}\right\|_{1,2} \leqslant C \varepsilon^{1 / 3}\left\|\hat{u}^{l}(t)\right\|_{2,2},
$$

where the constant $C$ is independent of $\varepsilon$.

Proof. - Indeed, since $N_{k}^{l}(y) \in C_{b}^{1}\left(\mathbb{R}^{3}\right)$ then, due to our choice of cut-off functions $\tau_{\varepsilon}$

$$
\begin{aligned}
& \left\|\nabla_{x}\left(\tilde{u}_{\varepsilon}^{l}-w_{\varepsilon}^{l}\right)\right\|_{1,2}^{2} \\
& \quad \leqslant \int_{\Omega}\left|\varepsilon \nabla_{x} \tau_{\varepsilon}\right|^{2}\left|\nabla_{x} \hat{u}^{l}(t)\right|^{2}+\left(1-\tau_{\varepsilon}\right)^{2}\left|\nabla_{y} N\left(\varepsilon^{-1} x\right)\right|^{2}\left|\nabla_{x} \hat{u}(t)\right|^{2}+C \varepsilon^{2}\left|\nabla_{x}^{2} \hat{u}(t)\right|^{2} \mathrm{~d} x \\
& \quad \leqslant C \int_{x \in \mathcal{O}_{\varepsilon}(\partial \Omega)}\left|\nabla_{x} \hat{u}^{l}(t)\right|^{2} \mathrm{~d} x+C \varepsilon^{2}\left\|\hat{u}^{l}(t)\right\|_{2,2}^{2} .
\end{aligned}
$$


Applying the Hölder inequality to the first term in the right-hand side of (4.15) and using the embedding $W^{2,2} \subset W^{1,6}$, we derive that

$$
\int_{x \in \mathcal{O}_{\varepsilon}(\partial \Omega)}\left|\nabla_{x} \hat{u}^{l}(t)\right|^{2} \mathrm{~d} x \leqslant\left|\mathcal{O}_{\varepsilon}(\partial \Omega)\right|^{2 / 3}\left\|\hat{u}^{l}(t)\right\|_{1,6}^{2} \leqslant C \varepsilon^{2 / 3}\left\|\hat{u}^{l}(t)\right\|_{2,2}^{2}
$$

(here we have used the fact that the volume of the $\varepsilon$-neighbourhood $\left|\mathcal{O}_{\varepsilon}(\partial \Omega)\right| \leqslant C \varepsilon$ because $\Omega$ is assumed to be smooth). Estimates (4.15) and (4.16) prove the lemma.

Now we are ready to complete the proof of the theorem. To this end, we introduce the function $v_{\varepsilon}(t):=u_{\varepsilon}(t)-\hat{u}(t)$. Then

$$
\partial_{t} v_{\varepsilon}^{l}(t)=\left[A_{\varepsilon}^{l} u_{\varepsilon}^{l}-A_{0}^{l} \hat{u}^{l}(t)\right]-\left[f^{l}\left(u_{\varepsilon}(t)\right)-f^{l}(\hat{u}(t))\right],\left.\quad v_{\varepsilon}^{l}\right|_{\partial \Omega}=0 .
$$

Let us take the inner product in $L^{2}(\Omega)$ of this equation with the function $u_{\varepsilon}^{l}(t)-w_{\varepsilon}^{l}(t)$ :

$$
\begin{aligned}
\int_{0}^{T}\left(\partial_{t} v_{\varepsilon}^{l},\left(u_{\varepsilon}^{l}-w_{\varepsilon}^{l}\right)\right) \mathrm{d} t= & \int_{0}^{T}\left(A_{\varepsilon}^{l} u_{\varepsilon}^{l}(t)-A_{0}^{l} \hat{u}^{l}(t), u_{\varepsilon}^{l}(t)-w_{\varepsilon}^{l}(t)\right) \mathrm{d} t \\
& -\int_{0}^{T}\left(f^{l}\left(u_{\varepsilon}(t)\right)-f^{l}(\hat{u}(t)), u_{\varepsilon}^{l}(t)-w_{\varepsilon}^{l}(t)\right) \mathrm{d} t .
\end{aligned}
$$

Note that definitions (4.8) and (4.13) imply the estimate

$$
\left\|u_{\varepsilon}^{l}(t)-w_{\varepsilon}^{l}(t)-v_{\varepsilon}^{l}(t)\right\|_{0,2} \leqslant C \varepsilon\left\|\hat{u}^{l}(t)\right\|_{1,2} .
$$

Consequently, estimate (1.17) and (4.3) imply the inequality

$$
\begin{aligned}
& \left|\int_{0}^{T}\left(\partial_{t} v_{\varepsilon}^{l}(t), u_{\varepsilon}^{l}(t)-w_{\varepsilon}^{l}(t)\right) \mathrm{d} t-\int_{0}^{T}\left(\partial_{t} v_{\varepsilon}^{l}(t), v_{\varepsilon}^{l}(t)\right) \mathrm{d} t\right| \\
& \quad \leqslant C \varepsilon\left(\int_{0}^{T}\left\|\partial_{t} v_{\varepsilon}^{l}(t)\right\|_{0,2}^{2}\right)^{1 / 2}\left(\int_{0}^{T}\left\|\hat{u}^{l}(t)\right\|_{1,2}^{2} \mathrm{~d} t\right)^{1 / 2} \leqslant Q\left(\left\|u_{0}\right\|_{L^{\infty} \cap W^{1,2}}\right) \varepsilon T,
\end{aligned}
$$

for a certain monotonic function $Q$ independent of $\varepsilon$.

The third term in (4.18) can be estimated analogously using the fact that we have the uniform with respect to $\varepsilon L^{\infty}$-estimate for the solutions $u_{\varepsilon}(t)$ and $\hat{u}(t)$ (due to Corollary 1.4):

$$
\begin{aligned}
& \mid \int_{0}^{T}\left(f^{l}\left(u_{\varepsilon}(t)\right)-f^{l}(\hat{u}(t)), u_{\varepsilon}^{l}(t)-w_{\varepsilon}^{l}(t)\right) \mathrm{d} t \\
& \quad-\int_{0}^{T}\left(f^{l}\left(u_{\varepsilon}(t)\right)-f^{l}(\hat{u}(t)), v_{\varepsilon}^{l}(t)\right) \mathrm{d} t \mid \leqslant Q\left(\left\|u_{0}\right\|_{0, \infty}\right) \varepsilon T,
\end{aligned}
$$

with the appropriate $Q$ independent of $\varepsilon$. 
Thus, it remains only to estimate the most complicated second term of (4.18). We will do so using the results of Lemmata 4.1 and 4.2 and the uniform ellipticity (with the constant $v>0$ ) of the operators $A_{\varepsilon}^{l}$ :

$$
\begin{aligned}
- & \left(A_{\varepsilon}^{l} u_{\varepsilon}^{l}(t)-A_{0}^{l} \hat{u}^{l}(t), u_{\varepsilon}^{l}-w_{\varepsilon}^{l}\right) \\
= & -\left(A_{\varepsilon}^{l}\left(u_{\varepsilon}^{l}(t)-\tilde{u}_{\varepsilon}^{l}(t)\right), u_{\varepsilon}^{l}(t)-w_{\varepsilon}^{l}(t)\right)-\left(A_{\varepsilon}^{l} \tilde{u}_{\varepsilon}^{l}(t)-A_{0}^{l} \hat{u}^{l}(t), u_{\varepsilon}^{l}(t)-w_{\varepsilon}^{l}(t)\right) \\
\geqslant & v\left\|u_{\varepsilon}^{l}(t)-\tilde{u}_{\varepsilon}^{l}(t)\right\|_{1,2}^{2}-C\left\|\tilde{u}_{\varepsilon}^{l}(t)-w_{\varepsilon}^{l}(t)\right\|_{1,2}^{2}-v / 4\left\|u_{\varepsilon}^{l}(t)-\tilde{u}_{\varepsilon}^{l}(t)\right\|_{1,2}^{2} \\
& -C \varepsilon^{2}\left\|\hat{u}^{l}(t)\right\|_{2,2}^{2}-v / 4\left\|u_{\varepsilon}^{l}(t)-w_{\varepsilon}^{l}(t)\right\|_{1,2}^{2} \geqslant-C_{1} \varepsilon^{2 / 3}\left\|\hat{u}^{l}(t)\right\|_{2,2}^{2} .
\end{aligned}
$$

Intergating inequalty (4.22) over $t \in[0, T]$ and taking into the account estimate (4.3), we have

$$
\int_{0}^{T}\left(A_{\varepsilon}^{l} u_{\varepsilon}^{l}(t)-A_{0} \hat{u}^{l}(t), u_{\varepsilon}^{l}(t)-w_{\varepsilon}^{l}(t)\right) \mathrm{d} t \leqslant Q\left(\left\|u_{0}\right\|_{L^{\infty} \cap W^{1,2}}\right) \varepsilon^{2 / 3} T .
$$

Inserting estimates (4.20), (4.21) and (4.23) in relation (4.18) and taking into the account the fact that $v_{\varepsilon}^{l}(0)=0$, we derive the estimate:

$$
1 / 2\left\|v_{\varepsilon}^{l}(T)\right\|_{0,2}^{2} \leqslant Q\left(\left\|u_{0}\right\|_{L_{\infty} \cap W^{1,2}}\right) \varepsilon^{2 / 3} T-\int_{0}^{T}\left(f^{l}\left(u_{\varepsilon}(t)\right)-f^{l}(\hat{u}(t)), v_{\varepsilon}^{l}(t)\right) \mathrm{d} t .
$$

Summing inequalities (4.24), for $l=1, \ldots, k$, we finally obtain

$$
\left\|v_{\varepsilon}(T)\right\|_{0,2}^{2} \leqslant 2 k Q \varepsilon^{2 / 3} T-2 \int_{0}^{T}\left(f\left(u_{\varepsilon}(t)\right)-f(\hat{u}(t)), v_{\varepsilon}(t)\right) \mathrm{d} t .
$$

Recall that the function $f \in C^{1}$ and we have the uniform with respect to $\varepsilon L^{\infty}$-estimates of solutions $u_{\varepsilon}(t)$ and $\hat{u}(t)$. That is why, we can estimate the intergal in (4.25) in a standard way and obtain the estimate

$$
\left\|v_{\varepsilon}(T)\right\|_{0,2}^{2} \leqslant 2 K \int_{0}^{T}\left\|v_{\varepsilon}(t)\right\|_{0,2}^{2} \mathrm{~d} t+2 k Q \varepsilon^{2 / 3} T
$$

where $K=K\left(\left\|u_{0}\right\|_{0, \infty}\right)$.

The Gronwall inequality applied to (4.26) finishes the proof of the theorem.

Remark 4.1. - Note that, in the case where the limit solution $\hat{u}(t)$ is smooth enough (e.g. $\hat{u} \in C^{1,2}\left(\Omega_{T}\right)$, it will be so for example if in addition $g \in C^{\beta}(\Omega)$ and the initial value $u_{0} \in C^{2+\beta}(\Omega)$ ), one can expect much better estimates than (4.2) (with exponent $1 / 2$ or even 1 instead of $1 / 3$ ). But, in this case, the function $Q$ will also depend on $\left\|u_{0}\right\|_{C^{2+\beta}}$ which is not permit to apply this result for estimation the difference between the attractors. So, keeping in mind the application of this estimate to the attractors, we 
cannot consider the initial data more regular than $C^{2 \beta} \cap W_{0}^{1,2}$ and this was the main difficulty in the proof of Theorem 4.1.

Now, having the error's estimate for approximation the individual solutions of $(0.1)$ by the solutions of homogenized equation (3.10), we are ready to derive the analogous error's estimates for the (global) attractors $\mathcal{A}^{\varepsilon}$ and $\mathcal{A}^{0}$. To this end, we need some additional information about the attractor $\mathcal{A}^{0}$ of the limit equation (3.10). Namely, we require the rate of convergence of images of bounded sets to the attractor $\mathcal{A}^{0}$ to be exponential, i.e.

$$
\operatorname{dist}_{L^{2}(\Omega)}\left(S_{t}^{0} B, \mathcal{A}^{0}\right) \leqslant Q\left(\|B\|_{\Phi}\right) \mathrm{e}^{-v t},
$$

for a certain positive exponent $v>0$ and the appropriate function $Q$.

THEOREM 4.2. - Let the assumptions of Theorem 4.1 hold and let the limit attractor $\mathcal{A}^{0}$ be exponential. Then, the nonsymmetric Hausdorff distance between $\mathcal{A}^{\varepsilon}$ and $\mathcal{A}^{0}$ possesses the following estimate

$$
\operatorname{dist}_{L^{2}(\Omega)}\left(\mathcal{A}^{\varepsilon}, \mathcal{A}^{0}\right) \leqslant C \varepsilon^{\kappa},
$$

where the constant $C$ and the exponent $0<\kappa<1$ can be computed explicitly.

Proof. - The assertion of the theorem is a simple corollary of estimates (4.2) and (4.27). Indeed, let $u_{\varepsilon}^{0} \in \mathcal{A}^{\varepsilon}$ be an arbitrary point of the attractor $\mathcal{A}^{\varepsilon}$. Then, due to (2.6), there is a complete bounded trajectory $u_{\varepsilon}(t) \in \mathcal{K}^{\varepsilon}$ such that $u_{\varepsilon}(0)=u_{\varepsilon}^{0}$. Moreover, according to estimate (2.12)

$$
\left\|u_{\varepsilon}(t)\right\|_{L^{\infty} \cap W^{1,2}(\Omega)} \leqslant C,
$$

where $C$ is independent of $t \in \mathbb{R}, \varepsilon$ and $u_{\varepsilon}^{0}$. Let us fix now an arbitrary $T \in \mathbb{R}_{+}$and consider the solution $\hat{u}(t)$ of the homogenized problem (3.10) with the initial value $\hat{u}(0):=u_{\varepsilon}(-T)$. Then, according to estimate (4.2),

$$
\left\|u_{\varepsilon}(0)-\hat{u}(T)\right\|_{0,2} \leqslant C_{1} \varepsilon^{1 / 3} \mathrm{e}^{K T},
$$

where the constants $C_{1}$ and $K$ depend only on $C$ from (4.29) and on the functions $Q$ and $K$ in (4.2) and are independent of $u_{\varepsilon}^{0}, T$ and $\varepsilon$.

From the other side, since the attractor $\mathcal{A}^{0}$ is exponential then

$$
\operatorname{dist}_{L^{2}(\Omega)}\left(\hat{u}(T), \mathcal{A}^{0}\right) \leqslant C_{2} \mathrm{e}^{-v T},
$$

where the constant $C_{2}$ is also independent of $u_{\varepsilon}^{0}, T$ and $\varepsilon$ (due to the uniform estimate (4.29)). Combining estimates (4.30) and (4.31), we derive that

$$
\operatorname{dist}_{L^{2}(\Omega)}\left(u_{\varepsilon}^{0}, \mathcal{A}^{0}\right) \leqslant C_{1} \varepsilon^{1 / 3} \mathrm{e}^{K T}+C_{2} \mathrm{e}^{-\nu T} .
$$

Recall that $T \geqslant 0$ is arbitrary, therefore we fix it in order to minimize the right-hand side of (4.33), i.e. satisfying the equation

$$
C_{1} \varepsilon^{1 / 3} \mathrm{e}^{K T}=C_{2} \mathrm{e}^{-v T} .
$$


Solving this equation and inserting $T=T(\varepsilon)$ in the right-hand side of (4.32), we obtain that

$$
\operatorname{dist}_{L^{2}(\Omega)}\left(u_{\varepsilon}^{0}, \mathcal{A}^{0}\right) \leqslant C_{3} \varepsilon^{\kappa},
$$

where $\kappa=\frac{v}{3(v+\kappa)}$. Since $u_{\varepsilon}^{0} \in \mathcal{A}^{\varepsilon}$ is arbitrary then (4.33) implies (4.28). Theorem 4.2 is proved.

COROLlaRY 4.1. - Let the assumptions of Theorem 4.2 hold. Then

$$
\operatorname{dist}_{C(\Omega)}\left(\mathcal{A}^{\varepsilon}, \mathcal{A}^{0}\right) \leqslant C \varepsilon^{\kappa_{1}},
$$

for the appropriate $0<\kappa_{1}<\kappa<1$.

Indeed, due to Corollary 2.1, the attractors $\mathcal{A}^{\varepsilon}$ and $\mathcal{A}^{0}$ are uniformly bounded in $C^{2 \beta^{\prime}}(\Omega), \beta>0$ therefore, estimate (4.27) together with the appropriate interpolation inequality implies (4.34).

Remark 4.2. - Assume, in addition, that the attractors $\mathcal{A}^{\varepsilon}, \varepsilon<\varepsilon_{0}$, are uniformly with respect to $\varepsilon$ exponential, i.e.

$$
\operatorname{dist}_{L^{2}(\Omega)}\left(S_{t}^{\varepsilon} B, \mathcal{A}^{\varepsilon}\right) \leqslant Q\left(\|B\|_{\Phi}\right) \mathrm{e}^{-v t},
$$

where the positive constant $v>0$ and the function $Q$ are independent of $\varepsilon$. Then, arguing as in the proof of Theorem 4.2, one can easily obtain the lower semicontinuity of the attractors $\mathcal{A}^{\varepsilon}$ and the estimate

$$
\operatorname{dist}_{L^{2}(\Omega)}\left(\mathcal{A}^{0}, \mathcal{A}^{\varepsilon}\right) \leqslant C \varepsilon^{\kappa}
$$

and, consequently, in this case we have estimate (4.2) not only for nonsymmetric Hausdorff distance but for the symmetric one as well.

Note in conclusion that there is a large class of systems of the form $(0.1)$ for which estimate (4.27) is known. Indeed, assume in addition that the nonlinear function $f$ has a gradient structure (which is always true in a scalar case $k=1$ )

$$
f(u)=\nabla_{u} F(u) .
$$

Then, as known (see e.g. [1]), Eq. (3.10) possesses a global Lyapunov function

$$
L(u)=\int_{\Omega} \sum_{i, j, l} \tilde{a}_{i j}^{l} \partial_{x_{i}} u^{l} \partial_{x_{j}} u^{l}+2 F(u)-2 g . u \mathrm{~d} x
$$

and, consequently, in the generic case, where we have only finite number of equilibria $\mathcal{R}:=\left\{z_{1}, \ldots, z_{N}\right\}$ for Eq. (3.10) and all of them are hyperbolic, the attractor of Eq. (3.10) is regular i.e. consists of a finite collection of the finite dimensional unstable manifolds $\mathcal{M}^{+}\left(z_{i}\right)$ of the equilibria points $z_{i} \in \mathcal{R}$ :

$$
\mathcal{A}^{0}=\bigcup_{i=1}^{N} \mathcal{M}^{+}\left(z_{i}\right)
$$


(see e.g. [1] or [15]). Moreover, it is also known (see [1]), that the regular attractor is exponential, i.e. (4.27) holds for the regular attractors. Thus, we have proved the following theorem.

THEOREM 4.3. - Let the assumptions of Theorem 4.2 holds and let in addition (4.37) be satisfied and all equilibria of the homogenized equation be hyperbolic. Then, the estimate (4.28) holds.

Let us consider now several examples of reaction-diffusion systems arising in mathematical physics for which the assumptions of Theorem 4.2 are fulfilled.

Example 4.1. - One of the simplest examples is a Chaffee-Infante equation in the non-homogenized almost-periodic media:

$$
\partial_{t} u=A_{\varepsilon} u-u^{3}+\alpha u+g(x),\left.\quad u\right|_{\partial \Omega}=0 .
$$

Here $u$ is a scalar function $(k=1), g \in L^{2}(\Omega), \alpha \in \mathbb{R}$ is a given constant and the operator $A_{\varepsilon}$ is defined via (0.3). All assumptions of Theorem 3.1 are evidently satisfied for this equation and, consequently, (4.40) possesses a global attractor $\mathcal{A}^{\varepsilon}$, for every $\varepsilon>0$. Moreover, these attractors tend to the limit attractor $\mathcal{A}^{0}$ of the homogenized problem as $\varepsilon \rightarrow 0$ in the sense of (3.11).

Note also that Eq. (4.40) has a gradient form ((4.37) is satisfied) and, consequently, for generic $g \in L^{2}(\Omega)$, the limit attractor $A_{0}$ is regular and exponential. Thus, in the case of periodic media estimate (4.28) is also valid for the external forces $g$ belonging to a certain open and dense set in $L^{2}(\Omega)$.

Example 4.2. - Consider now the following generalization of Lotka-Volterra system:

$$
\left\{\begin{array}{l}
\partial_{t} u_{i}=A_{\varepsilon}^{i} u_{i}-f_{i}\left(u_{i}\right)-u_{i}\left(\sum_{j=1}^{k} b_{i j} u_{j}^{2}\right)+g_{i}(x), \\
\left.u_{i}\right|_{\partial \Omega}=0, \quad i=1, \ldots, k
\end{array}\right.
$$

where $A_{\varepsilon}^{i}$ are defined via (0.3), $b_{i j} \geqslant 0$ are given nonnegative constants, $g_{i} \in L^{2}(\Omega)$ and the functions $f_{i}$ are assumed to satisfy the following assumptions:

$$
\begin{aligned}
& \begin{array}{l}
\text { 1. } f_{i} \in C^{1}(\mathbb{R}), \quad \text { 2. } f_{i}(v) \cdot \operatorname{sgn} v \geqslant 0 \quad \text { for }|v| \geqslant R, \\
\text { 3. }\left|f_{i}(v)\right| \leqslant C\left(1+|v|^{p}\right),
\end{array}
\end{aligned}
$$

for a certain constants $R>0, C>0$ and $p>0$. It is not difficult to verify that system (4.41) satisfies all assumptions of Theorem 3.1 (particularly, the anisotropic dissipativity condition (0.6) is valid with $p_{1}=p_{2}=\cdots=p_{k}=q$ for arbitrary $q>0$ ), consequently, the attractors $\mathcal{A}^{\varepsilon}, \varepsilon>0$ associated with the problems (4.41) converge as $\varepsilon \rightarrow 0$ to the attractor $\mathcal{A}^{0}$ of the limit homogenized problem.

Note also that, in the case where the matrix $\left\{b_{i j}\right\}_{i, j=1}^{k}$ is symmetric, i.e.

$$
b_{i j}=b_{j i} \geqslant 0, \quad i, j=1, \ldots, k,
$$


Eq. (4.41) has a gradient form, therefore, in the case of periodic media, estimate (4.28) for the rate of convergence of $\mathcal{A}^{\varepsilon}$ as $\varepsilon \rightarrow 0$ is valid for $g_{i}$ belonging to a certain open and dense subset of $L^{2}(\Omega)$ and for $b_{i j}$ satisfying (4.43).

Example 4.3. - We conclude our consideration by the so-called FitzHugh-Nagumo system:

$$
\begin{cases}\partial_{t} u_{1}=A_{\varepsilon}^{1} u_{1}-f\left(u_{1}\right)-u_{2}+g_{1}(x), & \left.u_{1}\right|_{\partial \Omega}=0, \\ \partial_{t} u_{2}=A_{\varepsilon}^{2} u_{2}+\delta u_{1}-\gamma u_{2}+g_{2}(x), & \left.u_{2}\right|_{\partial \Omega}=0 .\end{cases}
$$

Here $k=2$, the operators $A_{\varepsilon}^{i}$ are defined via $(0.3), \delta, \gamma>0$ are positive constants and the nonlinearity $f(v)$ is assumed to satisfy the fullowing assumptions:

$$
\text { 1. } f \in C^{1}(\Omega), \quad \text { 2. } f(v) \cdot v \geqslant-C+C_{1}|v|^{q+1}, \quad 3 .|f(u)| \leqslant C\left(1+|v|^{p}\right),
$$

for some positive $C, C_{1}, q, p>0$. A simple checking reveals that Eq. (4.44) satisfies all assumptions of Theorem 3.1 (particularly the anisotropic dissipativity assumption (0.6) holds with $p_{1}=p_{2}=r$ and arbitrary $r>0$ ), consequently the attractors $\mathcal{A}^{\varepsilon}$ associated with the nonhomogeneous problem (4.44) tend as $\varepsilon>0$ to the attractor $\mathcal{A}^{0}$ of the limit homogenized problem.

Note that Eq. (4.44) does not have a gradient structure. Nevertheless, it is shown in [4] that under the additional assumptions

$$
\text { 1. } f^{\prime}(v) \geqslant-\gamma \text { for all } v \in \mathbb{R},
$$

where $\gamma$ is the same as in the second equation of (4.44), this problem possesses a global Lyapunov function in the form:

$$
\begin{aligned}
L\left(u_{1}, u_{2}\right):= & \frac{1}{2}\left\|\partial_{t} u_{1}\right\|_{L^{2}}^{2}+\frac{1}{2 \delta}\left\|\partial_{t} u_{2}\right\|_{L^{2}}^{2}-\frac{\gamma}{2}\left(A_{\varepsilon}^{1} u_{1}, u_{1}\right)+\frac{\gamma}{2 \delta}\left(A_{\varepsilon}^{2} u_{2}, u_{2}\right) \\
& +\gamma F\left(u_{1}\right)+\gamma\left(u_{1}, u_{2}\right)-\frac{\gamma^{2}}{2 \delta}\|v\|_{L^{2}}^{2}-\gamma\left(g_{1}, u_{1}\right)+\frac{\gamma}{\delta}\left(g_{2}, u_{2}\right),
\end{aligned}
$$

where $(\cdot, \cdot)$ means the inner product in $L^{2}(\Omega), F(u)=\int_{0}^{u} f(u) \mathrm{d} u$ and the terms $\partial_{t} u_{1}$ and $\partial_{t} u_{2}$ should be expressed from equations (4.44) (according to Theorem 1.2 all terms in (4.47) are well posed on the attractor $\mathcal{A}^{\varepsilon}$ ). Thus, in the case of periodic media and under the additional assumption (4.47), estimate (4.28) holds for every $g_{i}$ from a dense and open subset of $L^{2}(\Omega)$.

Remark 4.3. - It is also known that the regular attractors are structurally stable in the sence, that representation (4.39) preserves under the small permutations of Eq. (3.10), moreover, the uiniform exponential attraction property is also valid if the pertrubation is small enough (see [1]). Note also that the non-homogenized problem (0.1) can be considered as a small pertrubation of the homogenized equation (3.10) because

$$
\left\|\left(A_{\varepsilon}\right)^{-1}-\left(A_{0}\right)^{-1}\right\|_{L^{2}(\Omega) \rightarrow L^{2}(\Omega)} \rightarrow 0 \quad \text { as } \varepsilon \rightarrow 0
$$

(see [20]). Therefore, one can expect that the uniform attraction property (4.35) is valid for our case if the limit homogenized attractor is regular. Then, according to Remark 4.2 we will have the lower semicontinuity of attractors $\mathcal{A}^{\varepsilon}$ as $\varepsilon=0$ and estimate (4.28) for 
the symmetric Hausdorff distance. We will give the rigorous proof of this assertion in the forthcoming paper.

\section{REFERENCES}

[1] A.V. Babin, M.I. Vishik, Attractors of Evolutionary Equations, North-Holland, Amsterdam, 1992.

[2] A. Bensoussan, J.-L. Lions, G. Papanicolaou, Asymptotic Analysis for Periodic Structures, North-Holland, Amsterdam, 1978.

[3] A. Bourgeat, L. Pankratov, Homogenization of semilinear parabolic equations in domains with spherical traps, Appl. Anal. 64 (3-4) (1997) 303-317.

[4] P. Freitas, C. Rocha, Lyapunov functional and stability for FitzHugh-Nagumo systems, JDE 169 (2001) 208-227.

[5] E. De Giorgi, S. Spagnolo, Sulla convergenza degli integrali dell'energia per operatori ellittici del secondo ordine, Boll. Unione Mat. Ital 8 (1973) 391-411.

[6] J. Hale, Asymptotic Behaviour of Dissipative Systems, in: Math. Surveys Monographs, Vol. 25, American Mathematical Society, Providence, RI, 1987.

[7] S. Kozlov, Averaging differential equations with almost-periodic rapidly oscillating coefficients, Math. USSR Sb. 35 (4) (1979) 481-498.

[8] Y. Kuznetsov, Elements of Applied Bifurkation Theory, in: Applied Math. Sciences, Vol. 112, Springer-Verlag, New York, 1995.

[9] O. Ladyzhenskaya, V. Solonnikov, N. Uraltseva, Linear and Quasilinear Parabolic Equations, Nauka, Moscow, 1967.

[10] B. Levitan, Almost-periodic Functions, Gostekhizdat, Moscow, 1953.

[11] B. Levitan, V. Zhikov, Almost-periodic Functions and Differential Equations, Moscow Univ. Press, Moscow, 1978.

[12] L. Pankratov, I. Chueshov, Homogenization of attractors of non-linear hyperbolic equations with asymptotically degenerate coefficients, Sb. Math. 190 (9) (1999) 1325-1352.

[13] R. Temam, Infinite Dimensional Dynamical Systems in Mechanics and Physics, SpringerVerlag, New York, 1988.

[14] H. Triebel, Interpolation Theory, Function Spaces, Differential Operators, North-Holland, Amsterdam, 1978.

[15] M. Vishik, Asymptotic Behaviour of Solutions of Evolutionary Equations, Cambridge Univ. Press, Cambridge, 1992.

[16] M. Vishik, Attractors of Equations Arising in Mathematical Physics with Rapidly Oscillating Terms and their Averadging, Talk on the Conference Dedicated to the 60th Birthday of R. Temam (Paris, Poitiers, 2000) (joint work with B. Fiedler).

[17] M. Vishik, S. Zelik, The regular attractor for a non-linear elliptic system in a cylindrical domain, Math. Sb. 190 (6) (1999) 803-834.

[18] S. Zelik, The attractor for a nonlinear reaction-diffusion system with a supercritical nonlinearity and it's dimension, Rend. Acad. Naz. Sci. XL Mem. Mat. Appl. 24 (2000) $1-25$.

[19] S. Zelik, The dynamics of fast non-autonomous travelling waves and homogenization, in: M. Carrive, et al. (Eds.), Actes des journées «Jeunes numériciens » en l'honneur du 60ème anniversaire du Professeur Roger Temam, Poitiers, France, Mars 9-10, 2000, PoitouCharentes : Atlantique, 2001, pp. 131-142 (English).

[20] V. Zhikov, S. Kozlov, O. Oleinik, Homogenization of differential operators and integral functionals, Springer-Verlag, Berlin, 1994. 